\title{
On the flows associated to selfadjoint operators on metric measure spaces
}

\section{The Anh Bui, Piero D'Ancona, Xuan Thinh Duong \& Detlef Müller}

\section{Mathematische Annalen}

ISSN 0025-5831

Math. Ann.

DOI 10.1007/s00208-019-01857-w



照 Springer 
Your article is protected by copyright and all rights are held exclusively by SpringerVerlag GmbH Germany, part of Springer Nature. This e-offprint is for personal use only and shall not be self-archived in electronic repositories. If you wish to self-archive your article, please use the accepted manuscript version for posting on your own website. You may further deposit the accepted manuscript version in any repository, provided it is only made publicly available 12 months after official publication or later and provided acknowledgement is given to the original source of publication and a link is inserted to the published article on Springer's website. The link must be accompanied by the following text: "The final publication is available at link.springer.com". 


\section{On the flows associated to selfadjoint operators on metric measure spaces}

\section{The Anh Bui ${ }^{1} \cdot$ Piero D'Ancona ${ }^{2}$ D $\cdot$ Xuan Thinh Duong ${ }^{1} \cdot$ Detlef Müller $^{3}$}

Received: 8 March 2018 / Revised: 29 May 2019

(c) Springer-Verlag GmbH Germany, part of Springer Nature 2019

\section{Abstract}

Let $X$ be a metric space with a doubling measure satisfying $\mu(B) \gtrsim r_{B}^{n}$ for any ball $B$ with any radius $r_{B}>0$. Let $L$ be a non negative selfadjoint operator on $L^{2}(X)$. We assume that $e^{-t L}$ satisfies a Gaussian upper bound and that the flow $e^{i t L}$ satisfies a typical $L^{1}-L^{\infty}$ dispersive estimate of the form

$$
\left\|e^{i t L}\right\|_{L^{1} \rightarrow L^{\infty}} \lesssim|t|^{-\frac{n}{2}}
$$

Then we prove a similar $L^{1}-L^{\infty}$ dispersive estimate for a general class of flows $e^{i t \phi(L)}$, with $\phi(r)$ of power type near 0 and near $\infty$. In the case of fractional powers $\phi(L)=L^{v}, v \in(0,1)$, we deduce dispersive estimates for $e^{i t L^{v}}$ with data in Sobolev, Besov or Hardy spaces $H_{L}^{p}$ with $p \in(0,1]$, associated to the operator $L$.

Mathematics Subject Classification 42B37 · 47D08

Communicated by Loukas Grafakos.

Piero D'Ancona

dancona@mat.uniroma1.it

The Anh Bui

the.bui@mq.edu.au; bt_anh80@yahoo.com

Xuan Thinh Duong

xuan.duong@mq.edu.au

Detlef Müller

mueller@math.uni-kiel.de

1 Department of Mathematics and Statistics, Macquarie University, Sydney, NSW 2109, Australia

2 Dipartimento di Matematica, Sapienza Università di Roma, Piazzale A. Moro 2, 00185 Rome, Italy

3 Mathematisches Seminar, C.-A.-Universität Kiel, Ludewig-Meyn-Strasse 4, 24118 Kiel, Germany 


\section{Contents}

1 Introduction

2 Preliminary results

2.1 Kernel estimates

2.2 Hardy spaces associated to operators

3 Dispersive estimates for $e^{i t \phi(L)}$

3.1 Subordination formulas and dispersive estimates for $e^{i t \phi(L)}$

3.2 A case study: dispersive estimates for fractional Schrödinger semigroups . . . . . . . . . . . . .

4 Some applications

4.1 Hermite operators

4.2 Twisted Laplacians

4.3 Laguerre operators

References

\section{Introduction}

Let $(X, d, \mu)$ be a metric space endowed with a nonnegative Borel measure $\mu$ satisfying the doubling condition: there exists a constant $C>0$ such that

$$
\mu(B(x, 2 r)) \leq C \mu(B(x, r))
$$

for all $x \in X, r>0$ and all balls $B(x, r):=\{y \in X: d(x, y)<r\}$. In this paper we shall assume in addition that

$$
\mu(B(x, r)) \gtrsim r^{n}
$$

for all $x \in X$ and $r>0$ and for some $n \geq 1$.

We note that the doubling property (1) yields a constant $D>0$ so that

$$
\mu(B(x, \lambda r)) \leq C \lambda^{D} \mu(B(x, r)),
$$

for all $\lambda \geq 1, x \in X$ and $r>0$; and that

$$
\mu(B(x, r)) \leq C\left(1+\frac{d(x, y)}{r}\right)^{\tilde{n}} \mu(B(y, r)),
$$

for all $x, y \in X$ and $r>0$.

The main question discussed here is the following. Suppose a selfadjoint operator $L$ on $L^{2}(X)$ satisfies an $L^{1}-L^{\infty}$ dispersive estimate of the form

$$
\left\|e^{i t L}\right\|_{L^{1} \rightarrow L^{\infty}} \lesssim|t|^{-a}
$$

This is frequently the case for many important operators, notably the Laplacian $L=$ $-\Delta$ and its potential perturbations. Under this assumption, is it possible to deduce similar estimates for the more general class of flows $e^{i t \phi(L)}$ :

$$
\left\|\psi(L) e^{i t \phi(L)}\right\|_{L^{1} \rightarrow L^{\infty}} \lesssim|t|^{-b} ?
$$


For instance, if we choose $\phi(L)=\sqrt{L}$, we are asking if a dispersive estimate for the wave flow $e^{i t \sqrt{L}}$ can be deduced directly from a corresponding estimate for the Schrödinger flow $e^{i t L}$. The possibility of such a reduction is suggested by a suitable subordination formula connecting the two flows (see [33]); the formula was applied in [15] to the Hermite operator and the twisted Laplacian. Our main goal here is to extend this idea to more general functions $\phi(L)$ with power-like behaviour near 0 and $\infty$, and to prove sharper estimates in terms of Sobolev, Hardy or Besov norms, appropriately defined. Of course, the interest in dispersive estimates is justified by their crucial role in a large number of important applications to linear and nonlinear evolution equations and in harmonic analysis.

We shall make the following assumptions on the selfadjoint nonnegative operator $L:$

(A1) The Schrödinger flow $e^{i t L}$ satisfies a dispersive estimate:

$$
\left\|e^{i t L}\right\|_{L^{1} \rightarrow L^{\infty}} \lesssim t^{-n / 2}, \quad t \in\left(0, T_{0}\right)
$$

where $T_{0} \in(0,+\infty]$.

(A2) The kernel $p_{t}(x, y)$ of $e^{-t L}$ admits a Gaussian upper bound: $\exists C, c>0$ such that for all $x, y \in X$ and $t>0$,

$$
\left|p_{t}(x, y)\right| \leq \frac{C}{\mu(B(x, \sqrt{t}))} \exp \left(-\frac{d(x, y)^{2}}{c t}\right) .
$$

Under (A1)-(A2), we can prove rather sharp pointwise decay estimates for the flow $e^{i t \phi(L)}$, provided $\phi(r) \sim r^{m_{1}}$ near 0 and $\phi(r) \sim r^{m_{2}}$ near $\infty, m_{1}, m_{2}>0$ (see the precise assumptions (H1), (H2) at the beginning of Sect. 3). Our general estimates for $e^{i t \phi(L)}$ are given in Theorem 3.3 for the low frequency regime and Theorem 3.4 for the high frequency regime.

We illustrate our results in the special, and particularly interesting case of fractional powers $\phi(L)=L^{v}, v \in(0,1)$ (see Theorems 3.7 and 3.9):

Theorem 1.1 Assume L satisfies $(A 1)$ and $(A 2)$, and let $v \in(0,1)$. Then we have:

(i) For $s>(1-v) n+v$,

$$
\left\|e^{i t L^{v}} f\right\|_{L^{\infty}} \lesssim|t|^{-\frac{n-1}{2}}\left\|(I+L)^{s / 2} f\right\|_{L^{1}}, \quad|t|<T_{0} .
$$

(ii) For $p \in(0,1)$ and $s=n(1 / p-v)+v$,

$$
\left\|e^{i t L^{v}} f\right\|_{L^{\infty}} \lesssim|t|^{-\frac{n-1}{2}}\left\|L^{s / 2} f\right\|_{H_{L}^{p},} \quad|t|<T_{0} .
$$

(iii) Moreover, for $s=(1-v) n+v$ we have

$$
\left\|e^{i t L^{v}} f\right\|_{L^{\infty}} \lesssim|t|^{-\frac{n-1}{2}}\|f\|_{\dot{B}_{1,1}^{s, L}}
$$


Here $H_{L}^{p}$ is the Hardy space associated to $L$, which can be defined via square functions or, equivalently, by atomic decompositions; see Sect. 2.2 for details. To our knowledge, the use of Hardy spaces with $p<1$ is novel in this context (recall however that Beals [5] proved dispersive estimates for $H^{1}$ data). The spaces $\dot{B}_{1,1}^{s, L}$ associated to $L$ are a generalization of classical Besov spaces to the setting of metric measure spaces, also new to our knowledge. Their construction and some properties are given at the end of Sect. 3. Note in particular that in some cases (e.g., $L=-\Delta+|x|^{2}$ ) we can prove that the new Besov spaces contain properly the standard ones with the same indices.

The decay rate $\sim|t|^{-\frac{n-1}{2}}$ appearing in the Theorem is independent of $v$ and may be surprising at first. However it is easy to check that one can not expect a better decay for general $L$; see Sect. 3.2 for a discussion of this phenomenon and related counterexamples. Note however that for $L=-\Delta$ on $L^{2}\left(\mathbb{R}^{n}\right)$ estimate (iii) reduces to

$$
\left\|e^{i t(-\Delta)^{1 / 2}}\right\| L^{\infty} \lesssim|t|^{-\frac{n-1}{2}}\|f\|_{\dot{B}_{1,1}^{\frac{n+1}{2}}}
$$

which is known to be sharp for the wave flow.

The plan of the paper is the following. In Sect. 2 we prove some preliminary kernel estimates and recall the definition of Hardy spaces associated to $L$, giving an equivalent atomic characterization. In Sect. 3 we prove the crucial subordination formulas, extending the earlier results in [33]; we then apply the formulas to prove general dispersive estimates for the flows $e^{i t \phi(L)}$. We also sharpen our results in the case of fractional powers, and in particular we define Besov spaces associated to the operator $L$. In the final Sect. 4 we examine some applications to concrete operators, namely Hermite and Laguerre operators and the twisted Laplacian, and in particular we improve both the rate of decay and the loss of derivatives compared with the similar estimates in [15].

\section{Preliminary results}

\subsection{Kernel estimates}

Let $L$ be a nonnegative, self-adjoint operator on $L^{2}(X)$ satisfying (A2). Denote by $E_{L}(\lambda)$ the spectral decomposition of $L$. Then by spectral theory, for any bounded Borel function $F:[0, \infty) \rightarrow \mathbb{C}$ we can define

$$
F(L)=\int_{0}^{\infty} F(\lambda) d E_{L}(\lambda)
$$

as a bounded operator on $L^{2}(X)$.

We have the following useful lemma.

Lemma 2.1 (a) Let $\varphi \in \mathscr{S}(\mathbb{R})$ be an even function. Then for any $N>0$ there exists $C$ such that 


$$
\left|K_{\varphi(t \sqrt{L})}(x, y)\right| \leq \frac{C}{\mu(B(x, t))+\mu(B(y, t))}\left(1+\frac{d(x, y)}{t}\right)^{-N},
$$

for all $t>0$ and $x, y \in X$.

(b) Let $\varphi \in \mathscr{S}(\mathbb{R})$ be an even function. Then for any $1 \leq p \leq q \leq \infty$ we have

$$
\|\varphi(t \sqrt{L})\|_{L^{p} \rightarrow L^{q}} \lesssim t^{-\left(\frac{n}{p}-\frac{n}{q}\right)}, \quad t>0
$$

Estimate (7) was proved in [9, Lemma 2.3] for $X=\mathbb{R}^{n}$. We now give a new proof for the general case. To do this, we need the following estimates.

Lemma 2.2 Let $\lambda>0$. Then we have:

(a) For any $N>0$ and $s=N+D+1 / 2$, there exits $C=C(N)$ so that

$$
|F(\lambda \sqrt{L})(x, y)| \leq \frac{C}{\sqrt{\mu(B(x, \lambda)) \mu(B(y, \lambda))}}\left(1+\frac{d(x, y)}{\lambda}\right)^{-N}\|F\|_{W_{s}^{2}}
$$

for all $x, y \in \mathbb{R}^{n}$ and all Borel functions supported in $[1 / 2,2]$.

(b) For any $N>0$ and $s=2(N+D+1)$ there exits $C=C(N)$ so that

$$
|F(\lambda \sqrt{L})(x, y)| \leq \frac{C}{\sqrt{\mu(B(x, \lambda)) \mu(B(y, \lambda))}}\left(1+\frac{d(x, y)}{\lambda}\right)^{-N}\|F\|_{W_{s}^{\infty}}
$$

for all smooth functions $F$ supported in $[0,2]$ with $F^{(2 v+1)}(0)=0$ for all $v \in \mathbb{N}$.

Proof (a) Set $G(x)=F(\sqrt{x}) e^{x}$. Then we have

$$
G\left(\lambda^{2} L\right) e^{-\lambda^{2} L}=\frac{1}{2 \pi} \int_{\mathbb{R}} e^{-\lambda^{2}(1-i \tau) L} \widehat{G}(\tau) d \tau
$$

where $\widehat{G}$ is the Fourier transform of $G$.

This, along with the fact that $F(\lambda \sqrt{L})=G\left(\lambda^{2} L\right) e^{-\lambda^{2} L}$, implies

$$
K_{F(\lambda \sqrt{L})}(x, y)=\frac{1}{2 \pi} \int_{\mathbb{R}} \widehat{G}(\tau) p_{\lambda^{2}(1-i \tau)}(x, y) d \tau
$$

Recalling [10, Lemma 4.1] we have

$$
\begin{aligned}
\left|p_{z}(x, y)\right| \leq & \frac{C}{\left[\mu\left(B\left(x, \sqrt{\frac{|z|}{\cos \theta}}\right)\right) \mu\left(B\left(y, \sqrt{\frac{|z|}{\cos \theta}}\right)\right)\right]^{1 / 2}} \\
& \times \exp \left(-c \frac{d(x, y)^{2}}{|z|} \cos \theta\right) \frac{1}{(\cos \theta)^{D}}
\end{aligned}
$$

for all $x, y \in X$ and $z \in \mathbb{C}_{+}=\{z \in \mathbb{C}: \Re z>0\}$ where $\theta=\arg z$. 
It follows that

$$
\begin{aligned}
\left|p_{\lambda^{2}(1-i \tau)}(x, y)\right| \leq & \frac{C}{\left[\mu\left(B\left(x, \lambda \sqrt{1+\tau^{2}}\right)\right) \mu\left(B\left(y, \lambda \sqrt{1+\tau^{2}}\right)\right)\right]^{1 / 2}} \\
& \times \exp \left[-c \frac{d(x, y)^{2}}{\lambda^{2}\left(1+\tau^{2}\right)}\right]\left(1+\tau^{2}\right)^{D / 2} \\
& \leq \frac{C}{[\mu(B(x, \lambda)) \mu(B(y, \lambda))]^{1 / 2}} \exp \left[-c \frac{d(x, y)^{2}}{\lambda^{2}\left(1+\tau^{2}\right)}\right](1+|\tau|)^{D} .
\end{aligned}
$$

This, along with (11), implies that

$$
\begin{aligned}
|F(\lambda \sqrt{L})(x, y)| \lesssim & |\widehat{G}(\tau)| \frac{1}{\sqrt{\mu(B(x, \lambda)) \mu(B(y, \lambda))}} \\
& \times \exp \left(-c \frac{d(x, y)^{2}}{\lambda^{2}\left(1+\tau^{2}\right)}\right)(1+|\tau|)^{D} d \tau \\
& \lesssim \frac{1}{\sqrt{\mu(B(x, \lambda)) \mu(B(y, \lambda))}}\left(1+\frac{d(x, y)}{\lambda}\right)^{-N} \\
& \int_{\mathbb{R}}|\widehat{G}(\tau)|(1+|\tau|)^{N+D} d \tau \\
& \lesssim \frac{1}{\sqrt{\mu(B(x, \lambda)) \mu(B(y, \lambda))}}\left(1+\frac{d(x, y)}{\lambda}\right)^{-N} \\
& \times\left(\int_{\mathbb{R}}|\widehat{G}(\tau)|\left(1+|\tau|^{2}\right)^{N+D+1} d \tau\right)^{1 / 2}\left(\int_{\mathbb{R}}\left(1+|\tau|^{2}\right)^{-1} d \tau\right)^{1 / 2} \\
& \lesssim \frac{1}{\sqrt{\mu(B(x, \lambda)) \mu(B(y, \lambda))}}\left(1+\frac{d(x, y)}{\lambda}\right)^{-N}\|\|_{W_{s}^{2}}
\end{aligned}
$$

where $s=N+D+1$.

Since supp $F \subset[1 / 2,2],\|G\|_{W_{s}^{2}} \sim\|F\|_{W_{s}^{2}}$. Hence, we obtain

$$
|F(\lambda \sqrt{L})(x, y)| \lesssim \frac{1}{\sqrt{\mu(B(x, \lambda)) \mu(B(y, \lambda))}}\left(1+\frac{d(x, y)}{\lambda}\right)^{-N}\|F\|_{W_{s}^{2}}
$$

(b) Since $F$ can be extended to be an even function, we can write $F(x)=f\left(x^{2}\right)$ for all $x \geq 0$ and $\|f\|_{W_{s}^{2}} \lesssim\|F\|_{W_{2 s}^{\infty}}$ for every $s>0$. See [40]. At this stage, arguing similarly to (a) we obtain (10).

We are ready to prove Lemma 2.1 .

Proof of Lemma 2.1 (a) Let $\psi_{0} \in C^{\infty}(\mathbb{R})$ supported in [0,2] such that $\psi_{0}=0$ on $[0,1]$ and $0 \leq \psi_{0} \leq 1$. Set $\psi(\lambda)=\psi_{0}(\lambda)-\psi_{0}(2 \lambda)$ and $\psi_{j}(\lambda)=\psi\left(2^{-j} \lambda\right)$ for $j \geq 1$. Then we have 


$$
\sum_{j \geq 0} \psi_{j}(\lambda)=1, \lambda>0
$$

Hence,

$$
\varphi(t \sqrt{L})=\sum_{j \geq 0} \psi_{j}(t \sqrt{L}) \varphi(t \sqrt{L})
$$

By (10) we have

$$
\left|\psi_{0}(t \sqrt{L}) \varphi(t \sqrt{L})(x, y)\right| \leq \frac{1}{\sqrt{\mu(B(x, \lambda)) \mu(B(y, \lambda))}}\left(1+\frac{d(x, y)}{\lambda}\right)^{-N}
$$

Since supp $\psi \subset[1 / 2,2]$, using (9) and (3), we have, for $j \geq 1$,

$$
\begin{aligned}
\left|\psi_{j}(t \sqrt{L}) \varphi(t \sqrt{L})(x, y)\right| \leq & \frac{C}{\sqrt{\mu\left(B\left(x, 2^{-j} t\right)\right) \mu\left(B\left(y, 2^{-j} t\right)\right)}} \\
& \times\left(1+\frac{d(x, y)}{2^{-j} t}\right)^{-N}\left\|h_{j}\right\|_{W_{s}^{2}} \\
\leq & \frac{C 2^{j D}}{\sqrt{\mu(B(x, t)) \mu(B(y, t))}}\left(1+\frac{d(x, y)}{t}\right)^{-N}\left\|h_{j}\right\|_{W_{s}^{2}}
\end{aligned}
$$

where $s=N+D+1$ and $h_{j}(\lambda)=\psi(\lambda) \varphi\left(2^{j} \lambda\right)$.

Since $\varphi \in \mathscr{S}(\mathbb{R}),\left\|h_{j}\right\|_{W_{s}^{2}} \leq C 2^{-j(D+1)}$. As a consequence,

$$
\left|\psi_{j}(t \sqrt{L}) \varphi(t \sqrt{L})(x, y)\right| \leq \frac{C 2^{-j}}{\sqrt{\mu(B(x, t)) \mu(B(y, t))}}\left(1+\frac{d(x, y)}{t}\right)^{-N}
$$

This, along with (12) and (13), implies that for each $N>0$ there exists $C$ such that

$$
|\varphi(t \sqrt{L})(x, y)| \leq \frac{C}{\sqrt{\mu(B(x, t)) \mu(B(y, t))}}\left(1+\frac{d(x, y)}{t}\right)^{-N}
$$

for all $x, y \in X$ and $t>0$.

Applying (4), we will obtain (7) as desired. This completes the proof of (a).

(b) From the estimate in part (a) and (2) we have

$$
\|\varphi(t \sqrt{L})\|_{L^{1} \rightarrow L^{\infty}} \lesssim t^{-n} \text { and }\|\varphi(t \sqrt{L})\|_{L^{1} \rightarrow L^{1}} \lesssim 1
$$

for all $t>0$. 
By interpolating, we have

$$
\|\varphi(t \sqrt{L})\|_{L^{1} \rightarrow L^{q}} \lesssim t^{-\left(n-\frac{n}{q}\right)}
$$

for all $t>0$ and $1 \leq q \leq \infty$.

On the other hand, from the estimate in part (a) again we have

$$
\|\varphi(t \sqrt{L})\|_{L^{q} \rightarrow L^{q}} \lesssim 1
$$

The last two estimates, in combination with interpolation, imply (8) as desired.

\subsection{Hardy spaces associated to operators}

We first recall from $[25,27]$ the definition of the Hardy spaces associated to an operator. Let $L$ be a nonnegative self-adjoint operator on $L^{2}(X)$ satisfying the Gaussian upper bound (A2). Let $0<p \leq 1$. Then the Hardy space $H_{L}^{p}(X)$ is defined as the completion of

$$
\left\{f \in L^{2}(X): S_{L} f \in L^{p}(X)\right\}
$$

under the norm $\|f\|_{H_{L}^{p}(X)}=\left\|S_{L} f\right\|_{L^{p}}$, where the square function $S_{L}$ is defined as

$$
S_{L} f(x)=\left(\int_{0}^{\infty} \int_{d(x, y)<t}\left|t^{2} L e^{-t^{2} L} f(y)\right|^{2} \frac{d \mu(y) d t}{\mu(B(x, t))}\right)^{1 / 2}
$$

Definition 2.3 (Molecules for $L$ ) Let $\epsilon>0,0<p \leq 1$ and $M \in \mathbb{N}$. A function $m(x)$ is called a $(p, 2, M, L, \epsilon)$-molecule associated to a ball $B \subset X$ of radius $r_{B}$ if there exists a function $b \in D\left(L^{M}\right)$ such that

(i) $m=L^{M} b$;

(ii) $\left\|L^{k} b\right\|_{L^{2}\left(S_{j}(B)\right)} \leq 2^{-j \epsilon} r_{B}^{2(M-k)} \mu\left(2^{j} B\right)^{1 / 2-1 / p}$ for all $k=0,1, \ldots, M$ and $j=$ $0,1,2 \ldots$

where $S_{j}(B)=2^{j} \backslash 2^{j-1} B$ as $j \geq 1$ and $S_{0}(B)=B$.

The definition of atoms is taken from [25,27] which requires (ii)-(iii). Assumption (ii) in particular can be thought of as a mild locality condition on the operator $L$.

Definition 2.4 (Hardy spaces associated to $L$ ) Given $\epsilon>0,0<p \leq 1$ and $M \in \mathbb{N}$, we say that $f=\sum \lambda_{j} m_{j}$ is a molecule $(p, 2, M, L, \epsilon)$-representation if $\left\{\lambda_{j}\right\}_{j=0}^{\infty} \in$ $\ell^{p}$, each $m_{j}$ is a $(p, 2, M, L, \epsilon)$-atom, and the sum converges in $L^{2}(X)$. The space $H_{L, \text { mol, } M, \epsilon}^{p}(X)$ is then defined as the completion of

$$
\left\{f \in L^{2}(X): f \text { has a molecule }(p, 2, M, L, \epsilon) \text {-representation }\right\}
$$


with the norm given by

$$
\begin{aligned}
\|f\|_{H_{L, \mathrm{~mol}, M, \epsilon}^{p}(X)}^{p} & \\
& =\inf \left\{\sum\left|\lambda_{j}\right|^{p}: f=\sum \lambda_{j} m_{j} \text { is a molecule }(p, 2, M, L, \epsilon) \text {-representation }\right\} .
\end{aligned}
$$

Theorem 2.5 [17] Let $\epsilon>0, p \in(0,1]$ and $M>\frac{n(2-p)}{4 p}$. Then the Hardy spaces $H_{L, \mathrm{~mol}, M, \epsilon}^{p}(X)$ and $H_{L}^{p}(X)$ coincide and have equivalent norms.

We note that if $L=-\Delta$ on $L^{2}\left(\mathbb{R}^{n}\right)$, then $H_{L}^{p}\left(\mathbb{R}^{n}\right)$ reduces to the standard Hardy space $H^{p}\left(\mathbb{R}^{n}\right)$ on $\mathbb{R}^{n}$ for $p \in(0,1]$. In general, depending on the choice of the operator $L$, it may happen that either $H^{p}\left(\mathbb{R}^{n}\right) \subset H_{L}^{p}\left(\mathbb{R}^{n}\right)$, or $H_{L}^{p}\left(\mathbb{R}^{n}\right) \subset H^{p}\left(\mathbb{R}^{n}\right)$, or $H^{p}\left(\mathbb{R}^{n}\right) \neq H_{L}^{p}\left(\mathbb{R}^{n}\right)$ without inclusions. See for example $[16,18]$. We examine now in more detail the case of the Hermite operator $L=-\Delta+|x|^{2}$ on $\mathbb{R}^{n}$, for which the Hardy spaces can be characterized via a new atomic decompositions as follows.

Let $\rho(x)=\min \left\{1,|x|^{-1}\right\}$ for $x \in \mathbb{R}^{n}$. Let $p \in(0,1]$. A function $a$ is called a $(p, \infty, \rho)$-atom associated to the ball $B\left(x_{0}, r\right)$ if

(i) $\operatorname{supp} a \subset B\left(x_{0}, r\right)$;

(ii) $\|a\|_{L^{\infty}} \leq\left|B\left(x_{0}, r\right)\right|^{-1 / p}$;

(iii) $\int x^{\alpha} a(x) d x=0$ for all $|\alpha| \leq\lfloor n(1 / p-1)\rfloor$ if $r<\rho\left(x_{0}\right) / 4$.

The Hardy space $H_{a t, \rho}^{p}\left(\mathbb{R}^{n}\right)$ is then defined as the set of all functions $f$ which can be expressed in the form $f=\sum_{j} \lambda_{j} a_{j}$ where $\left(\lambda_{j}\right)_{j} \in \ell^{p}$ and $a_{j}$ are $(p, \infty, \rho)$-atoms. Its norm is given by

$$
\|f\|_{H_{a t, \rho}^{p}\left(\mathbb{R}^{n}\right)}^{p}:=\inf \left\{\sum_{j}\left|\lambda_{j}\right|^{p}: f=\sum_{j} \lambda_{j} a_{j}\right\}
$$

where the infimum is taken over all possible atomic decompositions of $f$. From the definition, it is obvious that $H^{p}\left(\mathbb{R}^{n}\right) \varsubsetneqq H_{a t, \rho}^{p}\left(\mathbb{R}^{n}\right)$ for all $p \in(0,1]$; more importantly, we have $H_{a t, \rho}^{1}\left(\mathbb{R}^{n}\right) \equiv H_{L}^{1}\left(\mathbb{R}^{n}\right)$ (see for instance [20]), thus the Hardy space associated to the Hermite operator is larger than the standard one.

We recall an important result in [17, Proposition 3.27] which plays an important role in the sequel.

Proposition 2.6 Let $\epsilon>0, p \in(0,1]$ and $M>\frac{n(2-p)}{4 p}$. Suppose that $f=\sum_{j=1}^{N} \lambda_{j} a_{j}$ for some $N \in \mathbb{N}$, where $a_{j}^{\prime} s$ are $(p, 2,2 M, L, \epsilon)$ molecules and $\sum_{j=1}^{N}\left|\lambda_{j}\right|^{p}<\infty$. Then there exists a representation $f=\sum_{j=1}^{K} \gamma_{j} m_{j}$ for some $K \in \mathbb{N}$ where $m_{j}^{\prime}$ s are $(p, 2, M, L, \sigma)$ molecules for some $\sigma>0$ such that

$$
\sum_{j=1}^{K}\left|\gamma_{j}\right|^{p} \sim\|f\|_{H_{L}^{p}(X)}
$$




\section{Dispersive estimates for $e^{i t \phi(L)}$}

\subsection{Subordination formulas and dispersive estimates for $e^{i t \phi(L)}$}

Let $\phi: \mathbb{R}^{+} \rightarrow \mathbb{R}$ be a smooth function. We denote by (H1) and (H2) the following assumptions on $\phi$ :

(H1) There exists $0<m_{1} \leq 1$ such that

$$
\phi^{\prime}(r) \sim r^{m_{1}-1} \text { and }\left|\phi^{\prime \prime}(r)\right| \gtrsim r^{m_{1}-2}, \quad r \geq 1
$$

(H2) There exists $m_{2}>0$ such that

$$
\phi^{\prime}(r) \sim r^{m_{2}-1} \text { and }\left|\phi^{\prime \prime}(r)\right| \gtrsim r^{m_{2}-2}, \quad 0<r<1 .
$$

The following two results are crucial for the rest of the paper, and are strongly inspired by ideas from [33].

Theorem 3.1 Assume $\phi$ satisfies (H1) and $g$ is a $C^{\infty}$ function supported in $[1 / 2,2]$. Then there exist $c_{0}>1$, and functions and $\rho_{t}(x, \lambda)$ and $a_{t}(s, \lambda)$ defined on $\mathbb{R}^{2}$ for each $t$ satisfying

$$
\operatorname{supp} \rho_{t}(\cdot, \lambda) \subset\left[\lambda^{2} / 5,5 \lambda^{2}\right] \text { and }\left|\rho_{t}(x, \lambda)\right| \leq C\left(k,\|g\|_{C^{k}}, \phi\right)\left(t \lambda^{2 m_{1}}\right)^{-k}, k \geq 0 \text {, }
$$

and

$$
\operatorname{supp} a_{t}(\cdot, \lambda) \subset\left[2 c_{0}^{-1}, 2 c_{0}\right] \text { and }\left|a_{t}(s, \lambda)\right| \leq C\left(\|g\|_{C^{1}}, \phi\right) \text {, }
$$

so that

$$
g\left(\lambda^{-1} \sqrt{x}\right) e^{i t \phi(x)}=\rho_{t}(x, \lambda)+\sqrt{t \lambda^{2 m_{1}}} \eta\left(\lambda^{-2} x\right) \int e^{i x t \lambda^{2 m_{1}-2} s} a_{t}(s, \lambda) d s
$$

for all $x, t>0$ and $\lambda \geq 1$, where $\eta \in C^{\infty}(\mathbb{R})$ is supported in $[1 / 5,5]$ and $\eta \equiv 1$ on $[1 / 4,4]$.

Proof Let $k \in \mathbb{Z}$ and $t>0$. For $\lambda \geq 1$ we denote by $\Psi_{\lambda}(\xi)$ the Fourier transform of $g\left(\lambda^{-1} \sqrt{x}\right) e^{i t \phi(x)}$, i.e.,

$$
\begin{aligned}
\Psi_{\lambda}(\xi) & =\int g\left(\lambda^{-1} \sqrt{x}\right) e^{i t \phi(x)} e^{-i x \xi} d x \\
& =\lambda^{2} \int g(\sqrt{u}) e^{i\left[t \phi\left(\lambda^{2} u\right)-\lambda^{2} u \xi\right]} d u .
\end{aligned}
$$


Let $\tau \in C^{\infty}(\mathbb{R})$ supported in $\left[2 c_{0}^{-1}, 2 c_{0}\right]$ with $\tau \equiv 1$ in $\left[c_{0}^{-1}, c_{0}\right]$ where $c_{0}$ will be determined later. Then by the Fourier inversion formula we have

$$
\begin{aligned}
g\left(\lambda^{-1} \sqrt{x}\right) e^{i t \phi(x)}= & \eta\left(\lambda^{-2} x\right) \int\left(1-\tau\left(\frac{\xi}{t \lambda^{2 m_{1}-2}}\right)\right) \Psi_{\lambda}(\xi) e^{i \xi x} d \xi \\
& +\eta\left(\lambda^{-2} x\right) \int \tau\left(\frac{\xi}{t \lambda^{2 m_{1}-2}}\right) \Psi_{\lambda}(\xi) e^{i \xi x} d \xi \\
= & : \rho_{t}(x, \lambda)+A_{t}(x, \lambda)
\end{aligned}
$$

where $\eta \in C^{\infty}(\mathbb{R})$ is supported in $[1 / 5,5]$ and $\eta \equiv 1$ on $[1 / 4,4]$.

Observe that

$$
\partial_{u}\left[t \phi\left(\lambda^{2} u\right)-\lambda^{2} u \xi\right]=\lambda^{2} t \phi^{\prime}\left(\lambda^{2} u\right)-\lambda^{2} \xi
$$

We note that the integrand in the expression for $\rho_{t}(x, \lambda)$ is supported where either $\xi<c_{0}^{-1} t \lambda^{2 m_{1}-2}$ or $\xi>c_{0} t \lambda^{2 m_{1}-2}$. In this situation, by (H1) we can choose $c_{0}$ large enough so that

$$
\left|\partial_{u}\left[t \phi\left(\lambda^{2} u\right)-\lambda^{2} u \xi\right]\right| \gtrsim\left(\lambda^{2}|\xi|+t \lambda^{2 m_{1}}\right)
$$

Hence, by integration by parts in (17), we have for these $\xi$ that

$$
\left|\Psi_{\lambda}(\xi)\right| \leq C_{k, g, \phi} \lambda^{2}\left(\lambda^{2}|\xi|+t \lambda^{2 m_{1}}\right)^{-k}, \quad \forall k \geq 0, \lambda \geq 1
$$

This implies

$$
\left|\rho_{t}(x, \lambda)\right| \leq C_{k, g, \phi}\left(t \lambda^{2 m_{1}}\right)^{-k}, k \geq 0,
$$

which proves (14).

We now estimate the term $A_{t}(x, \lambda)$. By a change of variables, we have

$$
\begin{aligned}
A_{t}(x, \lambda) & =t \lambda^{2 m_{1}-2} \eta\left(\lambda^{-2} x\right) \int \tau(s) \Psi_{\lambda}\left(t \lambda^{2 m_{1}-2} s\right) e^{i x t \lambda^{2 m_{1}-2} s} d s \\
& =t \lambda^{2 m_{1}} \eta\left(\lambda^{-2} x\right) \int \tau(s) e^{i x t \lambda^{2 m_{1}-2} s} \int g(\sqrt{u}) e^{i\left[t \phi\left(\lambda^{2} u\right)-t \lambda^{2 m_{1}} u s\right]} d u d s \\
& =\sqrt{t \lambda^{2 m_{1}}} \eta\left(\lambda^{-2} x\right) \int e^{i x t \lambda^{2 m_{1}-2} s} a_{t}(s, \lambda) d s
\end{aligned}
$$

where

$$
a_{t}(s, \lambda)=\sqrt{t \lambda^{2 m_{1}}} \int \tau(s) g(\sqrt{u}) e^{i\left[t \phi\left(\lambda^{2} u\right)-t \lambda^{2 m_{1}} u s\right]} d u
$$


It is clear that $\operatorname{supp} a(\cdot, \lambda) \subset\left[2 c_{0}^{-1}, 2 c_{0}\right]$. Moreover, on the support of $g$ we have $1 / 4<u<4$. In this situation, by (H1) we have

$$
\left|\frac{\partial^{2}}{\partial u^{2}}\left[t \phi\left(\lambda^{2} u\right)-t \lambda^{2 m_{1}} u s\right]\right| \gtrsim t \lambda^{2 m_{1}} .
$$

Hence, by van der Corput's Lemma in [38] we obtain

$$
\left|a_{t}(x, \lambda)\right| \lesssim 1
$$

This implies $|a(s, \lambda)| \lesssim 1$ for all $s \in\left[2 c_{0}^{-1}, 2 c_{0}\right]$ and $\lambda \geq 1$. This proves (15).

In a similar way we have the following result:

Theorem 3.2 Assume $\phi$ satisfies (H2) and $g$ is a $C^{\infty}$ function supported in $[1 / 2,2]$. Then there exist $c_{0}>1$, and functions and $\rho_{t}(x, \lambda)$ and $a_{t}(s, \lambda)$ defined on $\mathbb{R}^{2}$ for each $t$ satisfying

$$
\left|\rho_{t}(x, \lambda)\right| \leq C\left(k,\|g\|_{C^{k}}, \phi\right)\left(t \lambda^{2 m_{2}}\right)^{-k}, \quad k \geq 0,
$$

and

$$
\operatorname{supp} a_{t}(\cdot, \lambda) \subset\left[2 c_{0}^{-1}, 2 c_{0}\right] \text { and }\left|a_{t}(s, \lambda)\right| \leq C\left(\|g\|_{C^{1}}, \phi\right) \text {. }
$$

so that

$$
g\left(\lambda^{-1} \sqrt{x}\right) e^{i t \phi(x)}=\rho_{t}(x, \lambda)+\sqrt{t \lambda^{2 m_{2}}} \eta\left(\lambda^{-2} x\right) \int e^{i x t \lambda^{2 m_{2}-2} s} a_{t}(s, \lambda) d s
$$

for all $x, t>0$ and $0<\lambda<1$, where $\eta \in C^{\infty}(\mathbb{R})$ is supported in $[5,1 / 5]$ and $\eta \equiv 1$ on $[1 / 4,4]$.

Proof The proof of this theorem is similar to that of Theorem 3.1 and we omit details.

We now apply the previous Theorems to obtain a high frequency (resp. low frequency) dispersive estimate for the flow $e^{i t \phi(L)}$ :

Theorem 3.3 Assume L satisfies (A1) and (A2), $\phi$ satisfies $(H 1)$, and $\psi \in C^{\infty}(\mathbb{R})$ is supported in $[1 / 2,2]$. Then we have

$$
\left|\psi\left(\lambda^{-1} \sqrt{L}\right) e^{i t \phi(L)} f\right| \lesssim|t|^{-\frac{n-1}{2}} \lambda^{\left(1-m_{1}\right) n+m_{1}}\|f\|_{L^{1}}, \quad \lambda \geq 1,|t|<T_{0} .
$$

Proof From Theorem 3.1 and spectral theory, there exist functions $\rho, a$ and $\eta$ as in Theorem 3.1 so that

$$
\begin{aligned}
\psi\left(\lambda^{-1} \sqrt{L}\right) e^{i t \phi(L)} & =\rho_{t}(L, \lambda)+\sqrt{t \lambda^{2 m_{1}}} \eta\left(\lambda^{-2} L\right) \int e^{i t \lambda^{2 m_{1}-2} s L} a_{t}(s, \lambda) d s \\
& =\rho_{t}(L, \lambda)+A_{t, \lambda}(L) .
\end{aligned}
$$


We first estimate the term related to $A_{t, \lambda}(L)$. By using (A1), (A2) and (15) we have

$$
\begin{aligned}
\left\|A_{t, \lambda}(L)\right\|_{L^{1} \rightarrow L^{\infty}} & \lesssim \sqrt{\lambda^{2 m_{1}} t}\left[\lambda^{2 m_{1}-2} t\right]^{-n / 2} \int_{2 c_{0}^{-1}}^{2 c_{0}} s^{-n / 2}\left|a_{t}(s, \lambda)\right| d s \\
& \lesssim t^{-\frac{n-1}{2}} \lambda^{\left(1-m_{1}\right) n+m_{1}} .
\end{aligned}
$$

We now take care of the term $\rho_{t}(L, \lambda)$. Let $\varphi \in C^{\infty}(\mathbb{R})$ with supp $\varphi \subset[1 / 6,6]$ and $\varphi \equiv 1$ in $[1 / 5,5]$. Since $\rho_{t}(\cdot, \lambda)$ is supported in $\left[\lambda^{2} / 5,5 \lambda^{2}\right]$, we have

$$
\rho_{t}(L, \lambda)=\varphi\left(\lambda^{-1} \sqrt{L}\right) \rho_{t}(L, \lambda) \varphi\left(\lambda^{-1} \sqrt{L}\right) .
$$

Therefore,

$$
\left\|\rho_{t}(L, \lambda)\right\|_{L^{1} \rightarrow L^{\infty}} \leq\left\|\varphi\left(\lambda^{-1} \sqrt{L}\right)\right\|_{L^{1} \rightarrow L^{2}}\left\|\rho_{t}(L, \lambda)\right\|_{L^{2} \rightarrow L^{2}}\left\|\varphi\left(\lambda^{-1} \sqrt{L}\right)\right\|_{L^{2} \rightarrow L^{\infty}}
$$

Using (8) we have

$$
\left\|\varphi\left(\lambda^{-1} \sqrt{L}\right)\right\|_{L^{1} \rightarrow L^{2}} \lesssim \lambda^{n / 2}, \quad \text { and } \quad\left\|\varphi\left(\lambda^{-1} \sqrt{L}\right)\right\|_{L^{2} \rightarrow L^{\infty}} \lesssim \lambda^{n / 2}
$$

By (14) we deduce

$$
\left\|\rho_{t}(L, \lambda)\right\|_{L^{2} \rightarrow L^{2}} \leq\left\|\rho_{t}(\cdot, \lambda)\right\|_{L^{\infty}} \lesssim\left(\lambda^{2 m_{1}} t\right)^{-\frac{n-1}{2}} .
$$

Therefore,

$$
\left\|\rho_{t}(L, \lambda)\right\|_{L^{1} \rightarrow L^{\infty}} \lesssim \lambda^{n}\left(\lambda^{2 m_{1}} t\right)^{-\frac{n-1}{2}}=t^{-\frac{n-1}{2}} \lambda^{\left(1-m_{1}\right) n+m_{1}} .
$$

Summing up, we have proved that

$$
\left\|\psi\left(\lambda^{-1} \sqrt{L}\right) e^{i t \phi(L)}\right\|_{L^{1} \rightarrow L^{\infty}} \lesssim t^{-\frac{n-1}{2}} \lambda^{\left(1-m_{1}\right) n+m_{1}}, \quad \lambda \geq 1 .
$$

This completes our proof.

For the low frequency estimate we have:

Theorem 3.4 Assume L satisfies (A1) and (A2), $\phi$ satisfies (H2), and $\psi \in C^{\infty}(\mathbb{R})$ is supported in $[1 / 2,2]$. Then we have

$$
\left|\psi\left(\lambda^{-1} \sqrt{L}\right) e^{i t \phi(L)} f\right| \lesssim|t|^{-\frac{n-1}{2}} \lambda^{\left(1-m_{2}\right) n+m_{2}}\|f\|_{L^{1}}, \quad 0<\lambda<1,|t|<T_{0}
$$

Proof If $T_{0}=\infty$, then we can argue similarly to the proof of Theorem 3.3. It remains to consider the case $T_{0}<\infty$. Arguing as for (21) we obtain

$$
\left|\psi\left(\lambda^{-1} \sqrt{L}\right) e^{i t \phi(L)} f\right| \lesssim \lambda^{n}\|f\|_{L^{1}}
$$


Now we note that

$$
\lambda^{n} \lesssim|t|^{-\frac{n-1}{2}} \lambda^{\left(1-m_{2}\right) n+m_{2}} \Longleftrightarrow|t| \lambda^{2 m_{2}} \lesssim 1
$$

and indeed we have

$$
|t| \lambda^{2 m_{2}} \leq T_{0} \cdot 1=T_{0}
$$

which concludes the proof.

Combining Theorems 3.3 and 3.4 we conclude:

Corollary 3.5 Let L satisfy $(A 1)$ and $(A 2)$ and let $\phi$ satisfy $(H 1)$ and $(H 2)$ with $m_{1}=m_{2}=m$. Assume that $\psi \in C^{\infty}(\mathbb{R})$ supported in $[1 / 2,2]$ then we have

$$
\left|\psi\left(\lambda^{-1} \sqrt{L}\right) e^{i t \phi(L)} f\right| \lesssim|t|^{-\frac{n-1}{2}} \lambda^{(1-m) n+m}\|f\|_{L^{1}}, \quad \lambda>0,|t|<T_{0}
$$

\subsection{A case study: dispersive estimates for fractional Schrödinger semigroups}

We consider in more detail the following special case of Corollary 3.5:

Proposition 3.6 Let L satisfy (A1) and (A2), and let $v \in(0,1)$. Assume that $\psi \in$ $C^{\infty}(\mathbb{R})$ is supported in $[1 / 2,2]$. Then we have

$$
\left|\psi\left(\lambda^{-1} \sqrt{L}\right) e^{i t L^{v}} f\right| \lesssim|t|^{-\frac{n-1}{2}} \lambda^{(1-v) n+v}\|f\|_{L^{1}}, \quad \lambda>0,|t|<T_{0} .
$$

Readers familiar with the stationary phase method may suspect that the estimate (25) is not sharp. Indeed, for the family of flows $e^{i t(-\Delta)^{v}}$ the decay rate is governed by the phase

$$
\phi(t, x, \xi)=x \cdot \xi+t|\xi|^{2 v}
$$

more precisely by the curvature of the surface $\nabla_{\xi} \phi=0$. In the case $v=1 / 2$, corresponding to the wave equation, the surface is degenerate and one gets a decay rate $\sim|t|^{-\frac{n-1}{2}}$, while for $v \neq 1 / 2$ one expects better decay, at least for suitable data. However, it is not difficult to check that for nonhomogeneous phases this simple picture is wrong: for arbitrary $v \in(0,1)$, one can construct operators $L$ such that $e^{i t L}$ has a decay rate $\sim t^{-\frac{n}{2}}$ while $e^{i t L^{v}}$ decays like $\sim t^{-\frac{n-1}{2}}$ at best in general. Thus, in this sense, our result is sharp.

We show the explicit construction for $v=1 / 3$. Define a piecewise $C^{1}$ function $a_{0}:[0,+\infty) \rightarrow[0,+\infty)$ as follows:

$$
a_{0}(s)=\left\{\begin{array}{ll}
s^{2} / 2 & \text { if } 0 \leq s \leq 1 / 2, \\
s^{3} & \text { if } 1 / 2 \leq s \leq 1, \\
3 s^{2}-2 & \text { if } s \geq 1
\end{array} \quad \text { so that } \quad a_{0}^{\prime}(s)= \begin{cases}s & \text { if } 0 \leq s \leq 1 / 2 \\
3 s^{2} & \text { if } 1 / 2 \leq s \leq 1 \\
6 s & \text { if } s \geq 1\end{cases}\right.
$$


Note that $a^{\prime}$ is strictly increasing with (positive) jumps at $s=1 / 2$ and $s=1$, and that for all $s \neq 1 / 2,1$ we have

$$
s \leq a_{0}^{\prime}(s) \leq 6 s, \quad 1 \leq a_{0}^{\prime \prime}(s) \leq 6
$$

We can obviously modify $a_{0}$ in two small neighbourhoods of the jump points and obtain a new $C^{\infty}$ function $a:[0,+\infty) \rightarrow[0,+\infty)$ such that for all $s \geq 0$

$$
s \leq a^{\prime}(s) \leq K s, \quad 1 \leq a^{\prime \prime}(s) \leq K
$$

for some positive constant $K>6$, and in addition

$$
a(s)= \begin{cases}s^{2} / 2 & \text { if } 0 \leq s \leq 1 / 2-\delta \\ s^{3} & \text { if } 1 / 2+\delta \leq s \leq 1-\delta, \quad \delta=1 / 100 . \\ 3 s^{2}-2 & \text { if } s \geq 1+\delta\end{cases}
$$

Note that we have also the symbol type property

$$
\left|a^{(j)}(s)\right| \lesssim s^{2-j}, \quad j=0,1,2, \ldots
$$

We then define the oscillatory integral with a radial phase

$$
I(t, x)=\int e^{i(x \cdot \xi+t \phi(\xi))} \psi(\xi) d \xi \quad \text { with } \phi(\xi)=a(|\xi|), \quad t \in \mathbb{R}, \quad x \in \mathbb{R}^{n}
$$

where $\psi \in C_{c}^{\infty}\left(\mathbb{R}^{n}\right)$ is a fixed test function; note that $\psi$ satisfies trivially the symbol property

$$
\left|\partial^{\alpha} \psi(\xi)\right| \lesssim|\xi|^{-|\alpha|}
$$

By the stationary phase method one gets the uniform estimate

$$
|I(t, x)| \leq C|t|^{-\frac{n}{2}}, \quad \forall t \neq 0, \quad x \in \mathbb{R}^{n}
$$

For instance, this is a special case of Theorem 3.1 in [26] (with the choices $m_{1}=$ $m_{2}=2, b_{1}=b_{2}=0$ so that $v_{1}=v_{2}=0$ ). The assumptions of the Theorem require, besides the symbol properties of $\phi, \psi$, that $\phi(\xi)$ satisfy

$$
|\nabla \psi| \simeq 1, \quad C_{1} \leq\left|\operatorname{det} D^{2} \phi\right| \leq C_{2}
$$

for some constants $C_{1}, C_{2}>0$; these conditions are easily verified from the above construction.

From estimate (26) one obtains immediately the dispersive estimate

$$
\left\|e^{i t L} f\right\|_{L^{\infty}} \lesssim|t|^{-\frac{n}{2}}\|f\|_{L^{1}}
$$


for the flow associated to the operator

$$
L=\phi(D) \text { i.e. } \quad L f=\mathcal{F}^{-1}(\phi(\xi) \widehat{f}) .
$$

Indeed, one can split $f$ in frequency on the ball $|\xi| \leq 10$, and to this first piece one applies (26); on the remaining piece, the operator $L$ coincides with $-3 \Delta-2$ and hence the estimate follows from the standard dispersive estimate for the Schrödinger equation.

However, the flow associated to $L^{1 / 3}$ can not decay faster than $|t|^{-\frac{n-1}{2}}$ in general. Indeed, for functions $f$ with

$$
\operatorname{supp} \widehat{f} \subset\{1 / 2+\delta<|\xi|<1-\delta\}
$$

we have

$$
L f=(-\Delta)^{3 / 2} f \quad \Longrightarrow \quad e^{i t L^{1 / 3}} f=e^{i t|D|} f
$$

so that $e^{i t L^{1 / 3}} f$ is precisely the wave flow applied to $f$, for which the sharp decay rate is known to be $\sim|t|^{-\frac{n-1}{2}}$.

It is clear that a similar construction can be performed for any power $L^{v}, v \in(0,1)$.

By a suitable frequency decomposition, we can deduce from 3.6 the following dispersive estimates with a loss of derivatives:

Theorem 3.7 Let L satisfy $(A 1)$ and $(A 2)$, and let $v \in(0,1)$.

(i) For $s>(1-v) n+v$,

$$
\left\|e^{i t L^{v}} f\right\|_{L^{\infty}} \lesssim|t|^{-\frac{n-1}{2}}\left\|(I+L)^{s / 2} f\right\|_{L^{1}}, \quad|t|<T_{0} .
$$

(ii) For $p \in(0,1)$ and $s=n(1 / p-v)+v$,

$$
\left\|e^{i t L^{v}} f\right\|_{L^{\infty}} \lesssim|t|^{-\frac{n-1}{2}}\left\|L^{s / 2} f\right\|_{H_{L}^{p}}, \quad|t|<T_{0} .
$$

Proof (i) Let $\varphi_{0} \in C^{\infty}[0, \infty)$ so that $\varphi_{0}(\lambda)=1$ for $\lambda \in[0,1]$ and $\operatorname{supp} \varphi_{0} \subset[0,2]$. Let $\varphi(\lambda)=\varphi_{0}(\lambda)-\varphi_{0}(2 \lambda)$ and set $\varphi_{j}(\lambda)=\varphi\left(2^{-j} \lambda\right), j \geq 1$. Then we have $\operatorname{supp} \varphi \subset[1 / 2,2]$ and

$$
\sum_{j \geq 0} \varphi_{j}(\lambda)=1, \quad \forall \lambda \geq 0
$$


so that

$$
\begin{aligned}
(I+L)^{-s / 2} & =\sum_{j \geq 0} \varphi_{j}(\sqrt{L})(I+L)^{-s / 2} \\
& =\sum_{j \geq 0} 2^{-j s}\left[2^{j s} \varphi_{j}(\sqrt{L})(I+L)^{-s / 2}\right] \\
& =: \sum_{j \geq 0} 2^{-j s} \widetilde{\varphi}_{j}(\sqrt{L})
\end{aligned}
$$

It follows that

$$
e^{i t L^{v}} f=\sum_{j \geq 0} 2^{-j s} \widetilde{\varphi}_{j}(\sqrt{L}) e^{i t L^{v}}(I+L)^{s / 2} f
$$

Hence,

$$
\begin{aligned}
\left\|e^{i t L^{v}} f\right\|_{L^{\infty}} & \leq \sum_{j \geq 0} 2^{-j s}\left\|\widetilde{\varphi}_{j}(\sqrt{L}) e^{i t L^{v}}(I+L)^{s / 2} f\right\|_{L^{\infty}} \\
& =\sum_{j \geq 0} I_{j}
\end{aligned}
$$

For $j \geq 1$, by applying Proposition 3.6 we have

$$
\begin{aligned}
\sum_{j \geq 1} I_{j} & \lesssim \sum_{j \geq 1} 2^{-j s}|t|^{-\frac{n-1}{2}} 2^{j[(1-v) n+v]}\left\|(I+L)^{s / 2} f\right\|_{L^{1}} \\
& \lesssim|t|^{-\frac{n-1}{2}}\left\|(I+L)^{s / 2} f\right\|_{L^{1}}
\end{aligned}
$$

as long as $s>(1-v) n+v$.

For $j=0$ we have

$$
\widetilde{\varphi}_{0}(\sqrt{L}) e^{i t L^{v}}(I+L)^{s / 2} f=\sum_{k \leq 1} \psi\left(2^{-k} \sqrt{L}\right) \widetilde{\varphi}_{0}(\sqrt{L}) e^{i t L^{v}}(I+L)^{s / 2} f
$$

where $\psi \in C_{0}^{\infty}(\mathbb{R})$ is a partition of unity function, i.e., $\psi \in \mathcal{S}(\mathbb{R})$ such that supp $\psi \subset$ $[1 / 2,2]$ and

$$
\sum_{j \in \mathbb{Z}} \psi\left(2^{-j} \lambda\right)=1 \text { on }(0, \infty)
$$

By applying Proposition 3.6 we have

$$
\begin{aligned}
I_{0} & \lesssim \sum_{k \leq 1}|t|^{-\frac{n-1}{2}} 2^{k[(1-v) n+v]}\left\|\widetilde{\varphi}_{0}(\sqrt{L})(I+L)^{s / 2} f\right\|_{L^{1}} \\
& \lesssim|t|^{-\frac{n-1}{2}}\left\|\widetilde{\varphi}_{0}(\sqrt{L})(I+L)^{s / 2} f\right\|_{L^{1}} .
\end{aligned}
$$


Since $\widetilde{\varphi}_{0}(\lambda)$ can be extended to be an even function in $\mathscr{S}(\mathbb{R})$, then by $(8)$ we have

$$
\left\|\widetilde{\varphi}_{0}(\sqrt{L})(I+L)^{s / 2} f\right\|_{L^{1}} \lesssim\left\|(I+L)^{s / 2} f\right\|_{L^{1}}
$$

As a consequence,

$$
I_{0} \lesssim|t|^{-\frac{n-1}{2}}\left\|(I+L)^{s / 2} f\right\|_{L^{1}}
$$

Therefore,

$$
\left\|e^{i t L^{v}} f\right\|_{L^{\infty}} \lesssim|t|^{-\frac{n-1}{2}}\left\|(I+L)^{s / 2} f\right\|_{L^{1}, s>(1-v) n+v .}
$$

(ii) Let $\left\{\psi\left(2^{-j} \cdot\right)\right\}_{j \in \mathbb{Z}}$ with supp $\psi \subset[1 / 2,2]$ be a partition of unity function on $\mathbb{R} \backslash\{0\}$. We then have

$$
\begin{aligned}
e^{i t L^{v}} f & =\sum_{j \in \mathbb{Z}} \psi\left(2^{-j} \sqrt{L}\right) e^{i t L^{v}} f \\
& =\sum_{j \in \mathbb{Z}} L^{-s / 2} \psi\left(2^{-j} \sqrt{L}\right) e^{i t L^{v}} L^{s / 2} f
\end{aligned}
$$

where $s=n(1 / p-v)+v$.

Let $L^{s / 2} f \in H_{L}^{p}(X) \cap L^{2}(X)$. Then, similarly to classical results [5,31], from Theorem 2.5 and Proposition 2.6 it suffices to prove (ii) for all $f$ such that $L^{s / 2} f$ has a finite molecule presentation, i.e.,

$$
L^{s / 2} f=\sum_{\ell=0}^{N} \lambda_{\ell} a_{\ell}
$$

for some $N \in \mathbb{N}$, where $a_{j}$ are $(p, 2, M, L, \epsilon)$ molecules with $\epsilon>0, M>\frac{n(2-p)}{2 p}$, and

$$
\left\|L^{s / 2} f\right\|_{H_{L}^{p}} \sim\left(\sum_{\ell=1}^{N}\left|\lambda_{\ell}\right|^{p}\right)^{1 / p} .
$$

From the molecule presentation of $L^{s / 2} f$ we have

$$
e^{i t L^{\nu}} f=\sum_{\ell=0}^{N} \lambda_{\ell} \sum_{j \in \mathbb{Z}} L^{-s / 2} \psi\left(2^{-j} \sqrt{L}\right) e^{i t L^{\nu}} a_{\ell}
$$

so that

$$
\left\|e^{i t L^{\nu}} f\right\|_{L^{\infty}} \leq \sum_{\ell=0}^{N}\left|\lambda_{\ell}\right| \sum_{j \in \mathbb{Z}}\left\|L^{-s / 2} \psi\left(2^{-j} \sqrt{L}\right) e^{i t L^{\nu}} a_{\ell}\right\|_{L^{\infty}} .
$$


We now claim that

$$
\sum_{j \in \mathbb{Z}}\left\|L^{-s / 2} \psi\left(2^{-j} \sqrt{L}\right) e^{i t L^{v}} a\right\|_{L^{\infty}} \lesssim|t|^{-\frac{n-1}{2}}
$$

for all $(p, 2, M, L, \epsilon)$ molecules with $M>\frac{n(2-p)}{2 p}$ and $\epsilon>0$.

Once (29) is proved, the estimate in (ii) follows immediately. Indeed, from (28) and (29), we have

$$
\left\|e^{i t L^{v}} f\right\|_{L^{\infty}} \lesssim|t|^{-\frac{n-1}{2}} \sum_{\ell=0}^{\infty}\left|\lambda_{\ell}\right| \leq|t|^{-\frac{n-1}{2}}\left(\sum_{\ell}\left|\lambda_{\ell}\right|^{p}\right)^{1 / p} \sim|t|^{-\frac{n-1}{2}}\left\|L^{s / 2} f\right\|_{H_{L}^{p}}
$$

which proves (ii).

So, it suffices to prove (29). To do this, suppose that $a$ is a $(p, 2, M, L, \epsilon)$ molecule associated to a ball $B$ with $M>\frac{n(2-p)}{2 p}$ and $\epsilon>0$. Then there exists $b \in D\left(L^{M}\right)$ so that $a=L^{M} b$ satisfies (i) and (ii) in Definition 2.3. We now split the sum on the left hand side of (29) into 2 parts as follows:

$$
\begin{aligned}
\sum_{j \in \mathbb{Z}}\left\|L^{-s / 2} \psi\left(2^{-j} \sqrt{L}\right) e^{i t L^{v}} a\right\|_{L^{\infty}} & =\sum_{j \geq-\log _{2} r_{B}}\left\|L^{-s / 2} \psi\left(2^{-j} \sqrt{L}\right) e^{i t L^{v}} a\right\|_{L^{\infty}} \\
& +\sum_{j<-\log _{2} r_{B}}\left\|L^{-s / 2} \psi\left(2^{-j} \sqrt{L}\right) e^{i t L^{v}} a\right\|_{L^{\infty}} \\
= & : E_{1}+E_{2} .
\end{aligned}
$$

Let us take care of $E_{1}$ first. To do this, we write

$$
\begin{aligned}
E_{1} & =\sum_{j \geq-\log _{2} r_{B}^{2}}\left\|L^{-s / 2} \psi\left(2^{-j} \sqrt{L}\right) e^{i t L^{v}} a\right\|_{L^{\infty}} \\
& =\sum_{j \geq-\log _{2} r_{B}} 2^{-j s}\left\|2^{j s} L^{-s / 2} \psi\left(2^{-j} \sqrt{L}\right) e^{i t L^{v}} a\right\|_{L^{\infty}} .
\end{aligned}
$$

Applying Proposition 3.6 we deduce that

$$
\begin{aligned}
E_{1} & \lesssim \sum_{j \geq-\log _{2} r_{B}} 2^{-j s} 2^{j[(1-v) n+v]}|t|^{-\frac{n-1}{2}}\|a\|_{L^{1}} \\
& \lesssim \sum_{j \geq-\log _{2} r_{B}} 2^{-j n(1 / p-1)}|t|^{-\frac{n-1}{2}}\|a\|_{L^{1}}
\end{aligned}
$$


From (ii) in Definition 2.3 and (2) we have

$$
\begin{aligned}
\|a\|_{L^{1}} & =\sum_{k=0}^{\infty}\|a\|_{L^{1}\left(S_{k}(B)\right)} \leq \sum_{k=0}^{\infty} \mu\left(2^{k} B\right)^{1 / 2}\|a\|_{L^{2}\left(S_{k}(B)\right)} \quad \text { (due to Hölder's inequality) } \\
& \leq \sum_{k=0}^{\infty} 2^{-k \epsilon} \mu\left(2^{k} B\right)^{1-1 / p} \\
& \leq \sum_{k=0}^{\infty} 2^{-k(\epsilon+n(1 / p-1))} r_{B}^{-n(1 / p-1)}
\end{aligned}
$$

which implies $\|a\|_{L^{1}} \lesssim r_{B}^{-n(1 / p-1)}$.

Therefore,

$$
\begin{aligned}
E_{1} & \lesssim \sum_{j \geq-\log _{2} r_{B}}\left[2^{-j} r_{B}^{-1}\right]^{n(1 / p-1)}|t|^{-\frac{n-1}{2}} \\
& \lesssim|t|^{-\frac{n-1}{2}} .
\end{aligned}
$$

For the term $E_{2}$ inserting $a=L^{M} b$ into the expression of $E_{2}$ we have

$$
\begin{aligned}
E_{2} & =\sum_{j<-\log _{2} r_{B}}\left\|L^{M-s / 2} \psi\left(2^{-j} \sqrt{L}\right) e^{i t L^{v}} b\right\|_{L^{\infty}} \\
& =\sum_{j<-\log _{2} r_{B}} 2^{j(2 M-s)}\left\|2^{-j(2 M-s)} L^{M-s / 2} \psi\left(2^{-j} \sqrt{L}\right) e^{i t L^{v}} b\right\|_{L^{\infty}} .
\end{aligned}
$$

By Proposition 3.6 we have

$$
\begin{aligned}
E_{2} & \lesssim \sum_{j<-\log _{2} r_{B}} 2^{j(2 M-s)} 2^{j[(1-v) n+v]}|t|^{-\frac{n-1}{2}}\|b\|_{L^{1}} \\
& \lesssim \sum_{j<-\log _{2} r_{B}} 2^{j[2 M-n(1 / p-1)]}|t|^{-\frac{n-1}{2}}\|b\|_{L^{1}} .
\end{aligned}
$$

On the other hand, arguing similarly to (30) we have

$$
\|b\|_{L^{1}} \lesssim r_{B}^{[2 M-n(1 / p-1)]}
$$

Therefore,

$$
\begin{aligned}
E_{2} & \lesssim \sum_{j<-\log _{2} r_{B}}\left[2^{j} r_{B}\right]^{[2 M-n(1 / p-1)]}|t|^{-\frac{n-1}{2}} \\
& \lesssim|t|^{-\frac{n-1}{2}}
\end{aligned}
$$

as long as $M>\frac{n(2-p)}{2 p}>\frac{n(1-p)}{2 p}$. 
From the estimates of $E_{1}$ and $E_{2}$ we obtain (29). This competes our proof.

Some comments on Theorem 3.7 are in order:

(i) An estimate like (i) in Theorem 3.7 was proved earlier in [15, Theorem 1.2] for the special case of the twisted Laplacian $L$ on $\mathbb{R}^{2 d}$, with $d \geq 2$. Note however that the estimate in [15] was

$$
\left\|e^{i t L^{v}} f\right\|_{L^{\infty}} \lesssim\left(|t|+|t|^{\frac{s-2 d}{2 v}}\right)\left\|L^{s / 2} f\right\|_{L^{1}}
$$

for $s$ in the range $2 d>s>2 d-2 \min \{v, 1-v\}$. Due to the spectral gap in this situation, the term $(I+L)^{s / 2}$ in (i) of Theorem 3.7 can be replaced by $L^{s / 2}$. Thus, even in this special case, estimate (i) in Theorem 3.7 improves (31) giving both a better range for $s$ and a better decay.

(ii) The estimate (ii) in Theorem 3.7 is new. To the best of our knowledge, this is the first $H_{L}^{p}-L^{\infty}$ dispersive estimate in the literature.

(iii) In the particular case when $L=-\Delta$, estimate (25) can be improved as follows: for all $v \in(0,1) \backslash\{1 / 2\}$,

$$
\left|\psi\left(\lambda^{-1} \sqrt{L}\right) e^{i t L^{v}} f\right| \lesssim|t|^{-\frac{n}{2}} \lambda^{(1-v) n}\|f\|_{L^{1}}, \quad \lambda>0,|t|<\infty .
$$

See for example [24, Theorem 1]. Thus, following the proof of Theorem 3.7, we obtain that for $v \in(0,1) \backslash\{1 / 2\}$

$$
\left\|e^{i t L^{v}} f\right\|_{L^{\infty}} \lesssim|t|^{-\frac{n}{2}}\left\|(I+L)^{s / 2} f\right\|_{L^{1}}, \quad s>n(1-v),
$$

and

$$
\left\|e^{i t L^{v}} f\right\|_{L^{\infty}} \lesssim|t|^{-\frac{n}{2}}\left\|L^{s / 2} f\right\|_{H^{p}}, p \in(0,1), s=n(1 / p-v),
$$

where $H^{p}$ is a classical Hardy space. To our knowledge, the estimates (33) and (34) are new.

We now focus on the special case $v=1 / 2$, which corresponds to the wave-type flow $e^{i t \sqrt{L}}$. Arguing similarly to Theorem 3.7 we obtain:

Theorem 3.8 Let L satisfy $(A 1)$ and (A2).

(i) For $s>\frac{n-1}{2}$, we have

$$
\left\|\frac{e^{i t \sqrt{L}}}{\sqrt{L}} f\right\|_{L^{\infty}} \lesssim|t|^{-\frac{n-1}{2}}\left\|(I+L)^{s / 2} f\right\|_{L^{1}}, \quad|t|<T_{0} .
$$

(ii) For $p \in(0,1)$ and $s=n\left(\frac{1}{p}-\frac{1}{2}\right)-\frac{1}{2}$, we have

$$
\left\|\frac{e^{i t \sqrt{L}}}{\sqrt{L}} f\right\|_{L^{\infty}} \lesssim|t|^{-\frac{n-1}{2}}\left\|L^{s / 2} f\right\|_{H_{L}^{p}}, \quad|t|<T_{0} .
$$


Note that the decay $|t|^{-\frac{n-1}{2}}$ in Theorem 3.8 is sharp, as shown by the well known dispersive estimates for the free wave equation corresponding to the choice $L=-\Delta$. For $L=-\Delta+V$ with small potentials $V \in \mathscr{S}\left(\mathbb{R}^{n}\right), n \geq 3$, it was proved in [5] (see also [30]) that

$$
\left\|\frac{e^{i t \sqrt{L}}}{\sqrt{L}} f\right\|_{L^{\infty}} \lesssim|t|^{-\frac{n-1}{2}}\left\|(I-\Delta)^{s / 2} f\right\|_{H^{1}}, s=\frac{n-1}{2} .
$$

We see that estimate (ii) in Theorem 3.8 is new for $0<p<1$ even when $L=-\Delta$.

We also mention that for Schrödinger operators $L=-\Delta+V$ our assumptions (A1), (A2) are known to hold for several classes of potentials $V(x)$ on $\mathbb{R}^{n}$, both locally and globally in time. Concerning the global in time case, heat kernel estimates (A2) hold in any dimension under very mild assumptions on $V$, e.g. for Kato class potentials (see e.g. $[14,36])$. Dispersive estimates for the Schrödinger propagator of the form (A1) are known for rather general classes of potentials (see [1,13,28,41,42]); sharp results are known only for dimension $n=1$ [13] and $n=3$ [4].

Note that Theorems 3.7 and 3.8 give dispersive estimates corresponding to $s>$ $n(1-v)+v$. In order to prove estimates for the critical case $s=n(1-v)+v$, we need a new version of homogeneous Besov spaces. We conclude this section by proving a version of dispersive estimates for initial data in Besov spaces generated by $L$. Fix a Littlewood-Paley dyadic partition of unity $\Psi=\left\{\psi_{j}\right\}_{j \in \mathbb{Z}}$ on $\mathbb{R}$, and define for all $s \in \mathbb{R}, 1 \leq p, q<\infty$ the Besov space $\dot{B}_{p, q}^{s, L}(X)$ as the completion of the set

$$
\left\{f \in L^{2}(X):\|f\|_{\dot{B}_{p, q}^{s, L}}<\infty\right\}
$$

for the norm $\|\cdot\|_{B_{p, q}^{s, L}}$ given by

$$
\|f\|_{\dot{B}_{p, q}^{s, L}}:=\left\{\sum_{j \in \mathbb{Z}}\left(2^{j s}\left\|\psi_{j}(\sqrt{L}) f\right\|_{L^{p}}\right)^{q}\right\}^{1 / q}
$$

We note that this definition is independent of the choice of $\Psi$. Indeed, suppose that $\Phi=\left\{\varphi_{k}\right\}_{k \in \mathbb{Z}}$ is another dyadic partition of unity. Then for $j \in \mathbb{Z}$ and $f \in L^{2}$ we have

$$
\psi_{j}(\sqrt{L}) f=\sum_{k=j-2}^{j+2} \psi_{j}(\sqrt{L}) \varphi_{k}(\sqrt{L}) f
$$

By (8) we have

$$
2^{j s}\left\|\psi_{j}(\sqrt{L}) f\right\|_{L^{p}} \lesssim \sum_{k=j-2}^{j+2} 2^{j s}\left\|\varphi_{k}(\sqrt{L}) f\right\|_{L^{p}} \sim \sum_{k=j-2}^{j+2} 2^{k s}\left\|\varphi_{k}(\sqrt{L}) f\right\|_{L^{p}}
$$


and this implies, as claimed,

$$
\left\{\sum_{j \in \mathbb{Z}}\left(2^{j s}\left\|\psi_{j}(\sqrt{L}) f\right\|_{L^{p}}\right)^{q}\right\}^{1 / q} \sim\left\{\sum_{j \in \mathbb{Z}}\left(2^{j s}\left\|\varphi_{j}(\sqrt{L}) f\right\|_{L^{p}}\right)^{q}\right\}^{1 / q}
$$

We can now prove our dispersive estimate for Besov initial data. Note that for wave type flows $e^{i t \sqrt{L}}$ we recover the sharp derivative loss $s=\frac{n-1}{2}$ which is well know for the wave equation:

Theorem 3.9 Let L satisfy $(A 1)$ and $(A 2)$, and let $v \in(0,1)$. Then we have

$$
\left\|e^{i t L^{v}} f\right\|_{L^{\infty}} \lesssim|t|^{-\frac{n-1}{2}}\|f\|_{\dot{B}_{1,1}^{(1-v) n+v, L} .}
$$

In the particular case $v=\frac{1}{2}$ we get

$$
\left\|\frac{e^{i t \sqrt{L}}}{\sqrt{L}} f\right\|_{L^{\infty}} \lesssim|t|^{-\frac{n-1}{2}}\|f\|_{\dot{B}_{1,1}^{\frac{n-1}{2}, L}}
$$

Proof Let $\Psi=\left\{\psi_{j}\right\}$ be a Littlewood-Paley partition of unity. Then we have

$$
\psi_{j}(\sqrt{L})=\sum_{k=j-2}^{j+2} \psi_{j}(\sqrt{L}) \psi_{k}(\sqrt{L})
$$

so that

$$
\psi_{j}(\sqrt{L}) e^{i t L^{v}} f=\sum_{k=j-2}^{j+2} \psi_{j}(\sqrt{L}) \psi_{k}(\sqrt{L}) e^{i t L^{v}} f
$$

This implies

$$
\begin{aligned}
& \left\|\psi_{j}(\sqrt{L}) e^{i t L^{v}} f\right\|_{L^{\infty}} \\
& \quad=\sum_{k=j-2}^{j+2}\left\|\psi_{k}(\sqrt{L}) e^{i t L^{v}} \psi_{j}(\sqrt{L}) f\right\|_{L^{\infty}} \\
& \quad \lesssim \sum_{k=j-2}^{j+2}|t|^{-\frac{n-1}{2}} 2^{k((1-v) n+v)}\left\|\psi_{j}(\sqrt{L}) f\right\|_{L^{1}} \sim|t|^{-\frac{n-1}{2}} 2^{j((1-v) n+v)}\left\|\psi_{j}(\sqrt{L}) f\right\|_{L^{1}}
\end{aligned}
$$


where we used (25) in the second inequality. As a consequence,

$$
\begin{aligned}
\left\|e^{i t L^{v}} f\right\|_{L^{\infty}} & \leq \sum_{j \in \mathbb{Z}}\left\|\psi_{j}(\sqrt{L}) e^{i t L^{v}} f\right\|_{L^{\infty}} \\
& \lesssim \sum_{j \in \mathbb{Z}}|t|^{-\frac{n-1}{2}} 2^{j((1-v) n+v)}\left\|\psi_{j}(\sqrt{L}) f\right\|_{L^{1}} \\
& \sim|t|^{-\frac{n-1}{2}}\|f\|_{\dot{B}_{1,1}^{(1-v) n+v, L}}
\end{aligned}
$$

which proves (37).

The proof of (38) can be done in the same manner, hence we omit details. This completes our proof.

\section{Some applications}

In this section we will apply the obtained results in Sect. 3 to study dispersive estimates for Hermite operators, twisted Laplacians and Laguerre operators. It is worth noticing that the list of applications is not exhaustive, since we just intend to show the generality of our theory. Apart from these applications, one can find more applications in other setting such as Schrödinger operators with smooth potentials.

The estimates in Theorems 3.7, 3.8 and 3.9 hold true for all operators below. Hence, we will not list all of them, but concentrate on some specific estimates.

\subsection{Hermite operators}

Let $L=-\Delta+|x|^{2}$ be the Hermite operator on $\mathbb{R}^{n}$ with $n \geq 1$. Let $p_{t}(x, y)$ denote the kernel of the semigroup $e^{-t L}$. It is clear that $p_{t}(x, y)$ enjoys the Gaussian upper bound (A2), and we have an explicit representation for the kernel $p_{t}(x, y)$ :

$$
p_{t}(x, y)=\frac{1}{\pi^{n / 2}}\left(\frac{e^{-2 t}}{1-e^{-4 t}}\right)^{n / 2} \exp \left(-\frac{1}{4} \frac{1+e^{-2 t}}{1-e^{-2 t}}|x-y|^{2}-\frac{1}{4} \frac{1-e^{-2 t}}{1+e^{-2 t}}|x+y|^{2}\right)
$$

for all $t>0$ and $x, y \in \mathbb{R}^{n}$. See for example [39].

It is well-known that for any $\delta>0$ there exists $C>0$ so that

$$
\left\|e^{i t L}\right\|_{L^{1} \rightarrow L^{\infty}} \leq \frac{C}{t^{n / 2}}, \quad|t|<\pi / 2-\delta
$$

Therefore, the Hermite operator $L$ satisfies the conditions (A1) and (A2).

As a consequence of Theorems 3.7 and 3.8, we have 
Proposition 4.1 Let $L=-\Delta+|x|^{2}$ be the Hermite operator on $\mathbb{R}^{n}$ with $n \geq 1$. Then we have

$$
\left\|e^{i t L^{v}} f\right\|_{L^{\infty}} \lesssim|t|^{-\frac{n-1}{2}}\left\|L^{s / 2} f\right\|_{H_{L}^{p}}, \quad|t|<\pi / 2-\delta
$$

for $p \in(0,1)$ and $s=n(1 / p-v)+v ;$ and

$$
\left\|\frac{e^{i t \sqrt{L}}}{\sqrt{L}} f\right\|_{L^{\infty}} \lesssim|t|^{-\frac{n-1}{2}}\left\|L^{s / 2} f\right\|_{H_{L}^{p}},|t|<\pi / 2-\delta
$$

for $p \in(0,1)$ and $s=n\left(\frac{1}{p}-\frac{1}{2}\right)-\frac{1}{2}$.

As mentioned in Sect. 2.2 that $H^{p}\left(\mathbb{R}^{n}\right) \varsubsetneqq H_{L}^{p}\left(\mathbb{R}^{n}\right)$, hence the estimate above is sharper than the following estimate:

$$
\left\|e^{i t L^{v}} f\right\|_{L^{\infty}} \lesssim|t|^{-\frac{n-1}{2}}\left\|L^{s / 2} f\right\|_{H^{p}}, \quad|t|<\pi / 2-\delta
$$

for $p \in(0,1)$ and $s=n(1 / p-v)+v ;$ and

$$
\left\|\frac{e^{i t \sqrt{L}}}{\sqrt{L}} f\right\|_{L^{\infty}} \lesssim|t|^{-\frac{n-1}{2}}\left\|L^{s / 2} f\right\|_{H^{p}}, \quad|t|<\pi / 2-\delta
$$

for $p \in(0,1)$ and $s=n\left(\frac{1}{p}-\frac{1}{2}\right)-\frac{1}{2}$.

Proposition 4.2 For each $0<s<2$ we have $\dot{B}_{1,1}^{s, L}\left(\mathbb{R}^{n}\right) \hookrightarrow \dot{B}_{1,1}^{s}\left(\mathbb{R}^{n}\right)$.

Proof Let $\Psi=\left\{\psi_{j}\right\}$ be a partition of unity, $\psi_{j}(s)=\psi\left(2^{-j} s\right)$. Then we have

$$
\psi\left(2^{-j} \sqrt{-\Delta}\right) f=\sum_{k \in \mathbb{Z}} \psi\left(2^{-j} \sqrt{-\Delta}\right) \psi\left(2^{-k} \sqrt{L}\right) f
$$

so that

$$
\sum_{j \in \mathbb{Z}} 2^{j s} \psi\left(2^{-j} \sqrt{-\Delta}\right) f=\sum_{j \in \mathbb{Z}} 2^{j s} \sum_{k \in \mathbb{Z}} \psi\left(2^{-j} \sqrt{-\Delta}\right) \psi\left(2^{-k} \sqrt{L}\right) f
$$

It follows that

$$
\begin{aligned}
\sum_{j \in \mathbb{Z}} 2^{j s}\left\|\psi\left(2^{-j} \sqrt{-\Delta}\right) f\right\|_{L^{1}} \leq & \sum_{j \in \mathbb{Z}} 2^{j s} \sum_{k \in \mathbb{Z}}\left\|\psi\left(2^{-j} \sqrt{-\Delta}\right) \psi\left(2^{-k} \sqrt{L}\right) f\right\|_{L^{1}} \\
\leq & \sum_{j \in \mathbb{Z}} 2^{j s} \sum_{k \geq j}\left\|\psi\left(2^{-j} \sqrt{-\Delta}\right) \psi\left(2^{-k} \sqrt{L}\right) f\right\|_{L^{1}} \\
& +\sum_{j \in \mathbb{Z}} 2^{j s} \sum_{k<j}\left\|\psi\left(2^{-j} \sqrt{-\Delta}\right) \psi\left(2^{-k} \sqrt{L}\right) f\right\|_{L^{1}} \\
= & : I_{1}+I_{2} .
\end{aligned}
$$


By using (8) we have

$$
\begin{aligned}
I_{1} & \lesssim \sum_{j \in \mathbb{Z}} \sum_{k \geq j} 2^{(j-k) s} 2^{k s}\left\|\psi\left(2^{-k} \sqrt{L}\right) f\right\|_{L^{1}} \\
& \lesssim \sum_{k \in \mathbb{Z}} 2^{k s}\left\|\psi\left(2^{-k} \sqrt{L}\right) f\right\|_{L^{1}}=\|f\|_{\dot{B}_{1,1}^{s, L}}
\end{aligned}
$$

To estimate the term $I_{2}$, by (8) we have

$$
\begin{aligned}
I_{2} & =\sum_{j \in \mathbb{Z}} 2^{j s} \sum_{k<j}\left\|(-\Delta)^{-1} \psi\left(2^{-j} \sqrt{-\Delta}\right)(-\Delta) L^{-1} L \psi\left(2^{-k} \sqrt{L}\right) f\right\|_{L^{1}} \\
\lesssim & \sum_{j \in \mathbb{Z}} 2^{j s} \sum_{k<j} 2^{-2 j}\left\|(-\Delta) L^{-1} L \psi\left(2^{-k} \sqrt{L}\right) f\right\|_{L^{1}} \\
\lesssim & \sum_{j \in \mathbb{Z}} 2^{j s} \sum_{k<j} 2^{-2 j}\left\|L \psi\left(2^{-k} \sqrt{L}\right) f\right\|_{L^{1}} \\
& +\sum_{j \in \mathbb{Z}} 2^{j s} \sum_{k<j} 2^{-2 j}\left\||x|^{2} L^{-1} L \psi\left(2^{-k} \sqrt{L}\right) f\right\|_{L^{1}} \\
\lesssim & \sum_{j \in \mathbb{Z}} 2^{j s} \sum_{k<j} 2^{-2(j-k)}\left\|\widetilde{\psi}\left(2^{-k} \sqrt{L}\right) f\right\|_{L^{1}} \\
& +\sum_{j \in \mathbb{Z}} 2^{j s} \sum_{k<j} 2^{-2(j-k)}\left\||x|^{2} L^{-1} \widetilde{\psi}\left(2^{-k} \sqrt{L}\right) f\right\|_{L^{1}}
\end{aligned}
$$

where $\widetilde{\psi}(x)=x^{2} \psi(x)$.

It was proved in [2] that $|x|^{2} L^{-1}$ is bounded on $L^{1}$. Hence,

$$
\begin{aligned}
I_{2} & \lesssim \sum_{j \in \mathbb{Z}} 2^{j s} \sum_{k<j} 2^{-2(j-k)}\left\|\widetilde{\psi}\left(2^{-k} \sqrt{L}\right) f\right\|_{L^{1}} \\
& =\sum_{j \in \mathbb{Z}} \sum_{k<j} 2^{-(2-s)(j-k)} 2^{k s}\left\|\widetilde{\psi}\left(2^{-k} \sqrt{L}\right) f\right\|_{L^{1}} \\
& \lesssim \sum_{k \in \mathbb{Z}} 2^{k s}\left\|\widetilde{\psi}\left(2^{-k} \sqrt{L}\right) f\right\|_{L^{1}}
\end{aligned}
$$

Arguing similarly to the proof of (36) we have

$$
\sum_{k \in \mathbb{Z}} 2^{k s}\left\|\widetilde{\psi}\left(2^{-k} \sqrt{L}\right) f\right\|_{L^{1}} \lesssim\|f\|_{\dot{B}_{1,1}^{s, L}}
$$

Hence, $I_{2} \lesssim\|f\|_{\dot{B}_{1,1}^{s, L}}$. This completes our proof.

Proposition 4.3 We have $\dot{B}_{1,1}^{0} \varsubsetneqq \dot{B}_{1,1}^{0, L}$. 
Proof We claim that if $\int_{0}^{\infty}\left\|t^{2} L e^{-t^{2} L} f\right\|_{L^{1}} t^{-1} d t<\infty$, then $f \in \dot{B}_{1,1}^{0, L}$ and

$$
\|f\|_{\dot{B}_{1,1}^{0, L}} \lesssim \int_{0}^{\infty}\left\|t^{2} L e^{-t^{2} L} f\right\|_{L^{1}} \frac{d t}{t}
$$

Indeed, let $\psi_{j}(s)=\psi\left(2^{-j} s\right)$ be a partition of unity. By functional calculus we have for $j \in \mathbb{Z}$

$$
\psi\left(2^{-j} \sqrt{L}\right) f=c \int_{0}^{\infty}\left(t^{2} L\right)^{2} e^{-t^{2} L} \psi\left(2^{-j} \sqrt{L}\right) f \frac{d t}{t}
$$

where $c=\left[\int_{0}^{\infty} z^{2} e^{-z} \frac{d z}{z}\right]^{-1}$. Hence

$$
\begin{aligned}
\sum_{j \in \mathbb{Z}}\left\|\psi\left(2^{-j} \sqrt{L}\right) f\right\| & \lesssim \sum_{j \in \mathbb{Z}} \sum_{k \in \mathbb{Z}} \int_{2^{-k-1}}^{2^{-k}}\left\|\left(t^{2} L\right)^{2} e^{-t^{2} L} \psi\left(2^{-j} \sqrt{L}\right) f\right\|_{L^{1}} \frac{d t}{t} \\
& \lesssim \sum_{j \in \mathbb{Z}} \sum_{k \geq j} \int_{2^{-k-1}}^{2^{-k}}\left\|\left(t^{2} L\right)^{2} e^{-t^{2} L} \psi\left(2^{-j} \sqrt{L}\right) f\right\|_{L^{1}} \frac{d t}{t} \\
& +\sum_{j \in \mathbb{Z}} \sum_{k<j} \int_{2^{-k-1}}^{2^{-k}}\left\|\left(t^{2} L\right)^{2} e^{-t^{2} L} \psi\left(2^{-j} \sqrt{L}\right) f\right\|_{L^{1}} \frac{d t}{t} \\
= & : I_{1}+I_{2} .
\end{aligned}
$$

For the term $I_{1}$ we have

$$
\begin{aligned}
I_{1} & \lesssim \sum_{j \in \mathbb{Z}} \sum_{k \geq j} \int_{2^{-k-1}}^{2^{-k}} t^{2} 2^{2 j}\left\|t^{2} L e^{-t^{2} L} \widetilde{\psi}\left(2^{-j} \sqrt{L}\right) f\right\|_{L^{1}} \frac{d t}{t} \\
& \lesssim \sum_{j \in \mathbb{Z}} \sum_{k \geq j} \int_{2^{-k-1}}^{2^{-k}} 2^{2(j-k)}\left\|t^{2} L e^{-t^{2} L} \widetilde{\psi}\left(2^{-j} \sqrt{L}\right) f\right\|_{L^{1}} \frac{d t}{t} \\
& \lesssim \sum_{k \in \mathbb{Z}} \int_{2^{-k-1}}^{2^{-k}}\left\|t^{2} L e^{-t^{2} L} f\right\|_{L^{1}} \frac{d t}{t}
\end{aligned}
$$

where $\widetilde{\psi}(x)=x^{2} \psi(x)$. Here we have applied (8). Thus

$$
I_{1} \lesssim \int_{0}^{\infty}\left\|t^{2} L e^{-t^{2} L} f\right\|_{L^{1}} \frac{d t}{t}
$$


Similarly, for $\bar{\psi}(x)=x^{-2} \psi(x)$, we have

$$
\begin{aligned}
I_{2} & \lesssim \sum_{j \in \mathbb{Z}} \sum_{k<j} \int_{2^{-k-1}}^{2^{-k}} t^{-2} 2^{-2 j}\left\|\left(t^{2} L\right)^{3} e^{-t^{2} L} \bar{\psi}\left(2^{-j} \sqrt{L}\right) f\right\|_{L^{1}} \frac{d t}{t} \\
& \lesssim \sum_{k \in \mathbb{Z}} \int_{2^{-k-1}}^{2^{-k}}\left\|\left(t^{2} L\right)^{3} e^{-t^{2} L} f\right\|_{L^{1}} \frac{d t}{t} .
\end{aligned}
$$

We can write

$$
\left(t^{2} L\right)^{3} e^{-t^{2} L} f=8\left(\frac{t^{2}}{2} L\right)^{2} e^{-\frac{t^{2}}{2} L}\left[\frac{t^{2}}{2} L e^{-\frac{t^{2}}{2} L} f\right]
$$

Since the kernel of $\left(\frac{t^{2}}{2} L\right)^{2} e^{-\frac{t^{2}}{2} L}$ satisfies the Gaussian upper bound (see for example [12]), we have

$$
\left\|\left(t^{2} L\right)^{3} e^{-t^{2} L} f\right\|_{L^{1}} \lesssim\left\|\frac{t^{2}}{2} L e^{-\frac{t^{2}}{2} L} f\right\|_{L^{1}}
$$

As a consequence,

$$
I_{2} \lesssim \sum_{k \in \mathbb{Z}} \int_{2^{-k-1}}^{2^{-k}}\left\|\frac{t^{2}}{2} L e^{-\frac{t^{2}}{2} L} f\right\|_{L^{1}} \frac{d t}{t} \lesssim \int_{0}^{\infty}\left\|t^{2} L e^{-t^{2} L} f\right\|_{L^{1}} \frac{d t}{t}
$$

This, along with (41) and (40), implies

$$
\sum_{j \in \mathbb{Z}}\left\|\psi\left(2^{-j} \sqrt{L}\right)\right\| \lesssim \int_{0}^{\infty}\left\|t^{2} L e^{-t^{2} L} f\right\|_{L^{1}} \frac{d t}{t}
$$

which proves the claim (39).

We now turn to prove $\dot{B}_{1,1}^{0} \varsubsetneqq \dot{B}_{1,1}^{0, L}$. Indeed, if $f \in \dot{B}_{1,1}^{0}$, it was proved in [8] that

$$
\int_{0}^{\infty}\left\|t^{2} L e^{-t^{2} L} f\right\|_{L^{1}} \frac{d t}{t} \lesssim\|f\|_{\dot{B}_{1,1}^{0}}
$$

Therefore, applying (39) we have $f \in \dot{B}_{1,1}^{0, L}$ and

$$
\|f\|_{\dot{B}_{1,1}^{0, L}} \lesssim \int_{0}^{\infty}\left\|t^{2} L e^{-t^{2} L} f\right\|_{L^{1}} \frac{d t}{t} \lesssim\|f\|_{\dot{B}_{1,1}^{0}}
$$

This completes the proof. 
We would like to illustrate the advantage of dispersive estimates in Theorem 3.9 and of the new Besov spaces. In our situation $L=-\Delta+|x|^{2}$, if $n=1$, estimate (38) becomes

$$
\left\|\frac{e^{i t \sqrt{L}}}{\sqrt{L}} f\right\|_{L^{\infty}} \lesssim|t|^{-\frac{n-1}{2}}\|f\|_{\dot{B}_{1,1}^{0, L}}
$$

In this situation, the estimate (42) is sharper than the following estimate

$$
\left\|\frac{e^{i t \sqrt{L}}}{\sqrt{L}} f\right\|_{L^{\infty}} \lesssim|t|^{-\frac{n-1}{2}}\|f\|_{\dot{B}_{1,1}^{0}},
$$

since by Proposition 4.3, $\dot{B}_{1,1}^{0} \subset \dot{B}_{1,1}^{0, L}$.

Although in the general case $n \geq 2$ it is not clear whether the following estimate holds true

$$
\left\|\frac{e^{i t \sqrt{L}}}{\sqrt{L}} f\right\|_{L^{\infty}} \lesssim|t|^{-\frac{n-1}{2}}\|f\|_{\dot{B}_{1,1}^{\frac{n-1}{2}}}
$$

for the particular case $n=2,3,4$ we can find the spaces $\dot{B}_{1,1}^{\frac{n-1}{2}, L} \subset \dot{B}_{1,1}^{\frac{n-1}{2}}$ (see Proposition 4.2) such that

$$
\left\|\frac{e^{i t \sqrt{L}}}{\sqrt{L}} f\right\|_{L^{\infty}} \lesssim|t|^{-\frac{n-1}{2}}\|f\|_{\dot{B}_{1,1}^{\frac{n-1}{2}, L}} .
$$

We do not know at present if there exists $f \in \dot{B}_{1,1}^{\frac{n-1}{2}}$ such that $\frac{e^{i t \sqrt{L}}}{\sqrt{L}} f \notin L^{\infty}$.

\subsection{Twisted Laplacians}

Consider the twisted Laplacian on $\mathbb{R}^{n}$ with $n=2 d, d \in \mathbb{N}$ :

$$
L=-\frac{1}{2} \sum_{j=1}^{d}\left[\left(\partial_{x_{j}}-i y_{j}\right)^{2}-\left(\partial_{y_{j}}+i x_{j}\right)^{2}\right] .
$$

It is well-known that the kernel $p_{t}(x, y)$ of $e^{-t L}$ admits a Gaussian upper bound

$$
\left|p_{t}(x, y)\right| \leq \frac{C}{t^{d}} \exp \left(-\frac{|x-y|^{2}}{c t}\right) .
$$

See [32]. 
Moreover, by continuation of the corresponding heat kernel in [32] we obtain

$$
\left\|e^{i t L}\right\|_{L^{1} \rightarrow L^{\infty}} \lesssim t^{-d}, \quad|t|<\pi / 2-\delta .
$$

Therefore, the twisted Laplacian $L$ satisfies (A1) and (A2). As a consequence, all of the estimates in Theorems 3.7, 3.8 and 3.9 hold true for the twisted Laplacian.

\subsection{Laguerre operators}

Consider the space $X=(0, \infty)^{n}$ equipped with the Euclidean distance $d$ and measure $\mu$ given by $d \mu(x)=d \mu_{1}(x) \ldots d \mu_{n}(x)$ where $d \mu_{k}=x_{k}^{2 \alpha_{k}+1} d x_{k}, \alpha_{k}>-1$ for $k=1, \ldots, n$.

It is easy to see that

$$
\mu(B(x, r)) \sim \prod_{k=1}^{n}\left(r+x_{k}\right)^{2 \alpha_{k}+1} r
$$

where $B(x, r)=\{y \in X:|x-y|<r\}$ is the ball centered in $x=\left(x_{1}, x_{2}, \ldots, x_{n}\right)$ with radius $r$. It follows that the measure $\mu$ satisfies the doubling condition (1). Moreover, if $\alpha_{k}>-1 / 2$ for all $k$, then we have

$$
\mu(B(x, r)) \gtrsim r^{N}, \quad N=2 n+\sum_{k=1}^{n} 2 \alpha_{k} \geq 1
$$

for all $x \in X$ and $r>0$.

In this section, we always assume that $\alpha_{k}>-1 / 2$ for all $k$.

For $m \in \mathbb{N}$ and $\alpha_{k}>-1 / 2, k=1,2, \ldots, n$, consider the Laguerre functions $\psi_{k}^{\alpha}$ which are defined by

$$
\psi_{m}^{\alpha_{k}}(x)=\left(\frac{2 m !}{\Gamma\left(m+\alpha_{k}+1\right)}\right)^{1 / 2} L_{m}^{\alpha_{k}}\left(x^{2}\right) e^{-x^{2} / 2}, x \in \mathbb{R},
$$

where $L_{m}^{\alpha_{k}}$ are the $m$-th Laguerre polynomials. See for example [29].

We set $\psi_{m}^{\alpha}(x)=\prod_{k=1}^{n} \psi_{m}^{\alpha_{k}}\left(x_{k}\right)$ for each $k=1,2, \ldots, n$ and $x=\left(x_{1}, x_{2}, \ldots, x_{n}\right)$. It is well known that the Laguerre functions form an orthonormal basis for $L^{2}(X, d \mu)$. We now consider the Laguerre operator $L$, defined by

$$
L=-\Delta-\sum_{k=1}^{n} \frac{2 \alpha_{k}+1}{x_{k}} \frac{d}{d x_{k}}+|x|^{2}
$$

It is well known that

$$
L \psi_{m}^{\alpha}=\lambda_{m, \alpha} \psi_{m}^{\alpha}, \quad \lambda_{m, \alpha}=(4 m+2) n+\sum_{k=1}^{n} 2 \alpha_{k}
$$


Then the operator $L$ has a non-negative self-adjoint extension which is still denoted by $L$ with domain

$$
D(L)=\left\{f \in L^{2}(X, d \mu): \sum_{m} \lambda_{m, \alpha}^{2}\left|\left\langle f, \psi_{m}^{\alpha}\right\rangle_{d \mu}\right|^{2}<\infty\right\}
$$

Moreover, the heat kernel $p_{t}(x, y)$ associated to the semigroup $e^{-t L}$ is given by

$$
\begin{aligned}
p_{t}(x, y)= & \prod_{k=1}^{n} \frac{2 e^{-2 t}}{1-e^{-4 t}} \\
& \times \exp \left(-\frac{1}{2} \frac{1+e^{-4 t}}{1-e^{-4 t}}\left(x_{k}^{2}+y_{k}^{2}\right)\right)\left(x_{k} y_{k}\right)^{-\alpha_{k}} I_{\alpha_{k}}\left(\frac{2 e^{-2 t}}{1-e^{-4 t}} x_{k} y_{k}\right),
\end{aligned}
$$

for all $t>0, x, y \in X$ and $I_{\alpha_{k}}$ being the Bessel function. See for example [29].

Theorem 4.4 Let $\alpha_{k}>-1 / 2$ for all $k=1, \ldots, n$ and let $L$ be the Laguerre operator defined by (45). Then we have

$$
0<p_{t}(x, y) \lesssim \frac{C}{\mu(B(x, \sqrt{t}))} \exp \left(-\frac{|x-y|^{2}}{c t}\right)
$$

for all $x, y \in X$ and $t>0$.

Moreover,

$$
\left\|e^{i t L}\right\|_{L^{1} \rightarrow L^{\infty}} \lesssim t^{-N / 2}, \quad|t|<\pi / 2-\delta
$$

where $N=2 n+\sum_{k=1}^{n} 2 \alpha_{k}$.

Hence, the Laguerre operator satisfies (A1) and (A2).

Proof We refer to [6, Lemma 3.1] for the proof of the Gaussian upper bounds (46) and [37, Lemma 3.5] for the proof of the dispersive estimate (48).

Definition 4.5 Let $p \in\left(\frac{N}{N+1}, 1\right]$. A function $a$ is called a $p$-atom associated to the ball $B$ if

(i) $\operatorname{supp} a \subset B$

(ii) $\|a\|_{L^{\infty}(X)} \leq \mu(B)^{-1 / p}$

(iii) $\int a(x) d \mu(x)=0$.

To define the Hardy space $H_{C W}^{p}$ for $p$ below 1 , we need to introduce the Lipschitz spaces $\mathfrak{L}_{\alpha}$. We say that the function $f \in \mathfrak{L}_{\alpha}$ if there exists a constant $c>0$, such that

$$
|f(x)-f(y)| \leq c|B|^{\alpha}
$$

for all ball $B$ and $x, y \in B$, and the best constant $c$ can be taken to be the norm of $f$ and is denoted by $\|f\|_{\mathfrak{L}_{\alpha}}$. 
Definition 4.6 (Hardy spaces of Coifman and Weiss) Let $\frac{N}{N+1}<p \leq 1$. We say that a function $f \in H_{C W}^{p}(X)$ if $f \in L^{1}(X)$ for $p=1$, or $f \in \mathfrak{L}_{1 / p-1}^{*}$ for $p<1$, and there exists a sequence $\left(\lambda_{j}\right)_{j \in \mathbb{N}} \in \ell^{p}$ and a sequence of $p$-atoms $\left(a_{j}\right)_{j \in \mathbb{N}}$ such that $f=\sum_{j} \lambda_{j} a_{j}$ in $L^{1}(X)$ for $p=1$, and $f=\sum_{j} \lambda_{j} a_{j}$ in $\mathfrak{L}_{1 / p-1}^{*}$ for $p<1$. We set

$$
\|f\|_{H_{C W}^{p}}=\inf \left\{\left(\sum_{j}\left|\lambda_{j}\right|^{p}\right)^{1 / p}: f=\sum_{j} \lambda_{j} a_{j}\right\} .
$$

From Theorems 3.7 and 3.8, we have

Proposition 4.7 Let $\alpha_{k}>-1 / 2$ for all $k=1, \ldots, n$ and let $L$ be the Laguerre operator defined by (45). Then we have

$$
\left\|e^{i t L^{v}} f\right\|_{L^{\infty}} \lesssim|t|^{-\frac{N-1}{2}}\left\|L^{s / 2} f\right\|_{H_{L}^{p}}, \quad|t|<\pi / 2-\delta
$$

for $p \in(0,1)$ and $s=N(1 / p-v)+v ;$ and

$$
\left\|\frac{e^{i t \sqrt{L}}}{\sqrt{L}} f\right\|_{L^{\infty}} \lesssim|t|^{-\frac{N-1}{2}}\left\|L^{s / 2} f\right\|_{H_{L}^{p}},|t|<\pi / 2-\delta
$$

for $p \in(0,1)$ and $s=N\left(\frac{1}{p}-\frac{1}{2}\right)-\frac{1}{2}$.

From [7, Theorem 6.6] we have $H^{p}\left(\mathbb{R}^{n}\right) \varsubsetneqq H_{L}^{p}\left(\mathbb{R}^{n}\right)$ for $\frac{N}{N+1}<p \leq 1$. Hence the estimates above are sharper than the following estimates:

$$
\left\|e^{i t L^{\nu}} f\right\|_{L^{\infty}} \lesssim|t|^{-\frac{N-1}{2}}\left\|L^{s / 2} f\right\|_{H_{C W}^{p}}, \quad|t|<\pi / 2-\delta
$$

for $p \in\left(\frac{N}{N+1}, 1\right)$ and $s=N(1 / p-v)+v ;$ and

$$
\left\|\frac{e^{i t \sqrt{L}}}{\sqrt{L}} f\right\|_{L^{\infty}} \lesssim|t|^{-\frac{N-1}{2}}\left\|L^{s / 2} f\right\|_{H_{C W}^{p}}, \quad|t|<\pi / 2-\delta
$$

for $p \in\left(\frac{N}{N+1}, 1\right)$ and $s=N\left(\frac{1}{p}-\frac{1}{2}\right)-\frac{1}{2}$.

Acknowledgements X. T. Duong was supported by the Research Grant ARC DP160100153 from the Australian Research Council.

\section{References}

1. Artbazar, G., Yajima, K.: The $L^{p}$-continuity of wave operators for one dimensional Schrödinger operators. J. Math. Sci. Univ. Tokyo 7, 221-240 (2000)

2. Auscher, P., Ali, B.: Maximal inequalities and Riesz transform estimates on $L^{p}$ spaces for Schrödinger operators with nonnegative potentials. Ann. Inst. Fourier (Grenoble) 57(6), 1975-2013 (2007) 
3. Beals, M.: Optimal $L^{\infty}$ decay for solutions to the wave equation with a potential. Commun. Partial Differ. Equ. 19, 1319-1369 (1994)

4. Beceanu, M., Goldberg, M.: Schrödinger dispersive estimates for a scaling-critical class of potentials. Commun. Math. Phys. 314, 471-481 (2012)

5. Bownik, M.: Boundedness of operators on Hardy spaces via atomic decomposition. Proc. Am. Math. Soc. 133, 3535-3542 (2005)

6. Bui, T.A., Duong, X.T.: Laguerre operator and its associated weighted Besov and Triebel-Lizorkin spaces. Trans. Am. Math. Soc. 369(3), 2109-2150 (2017)

7. Bui, T.A., Duong, X.T., Ly, F.K.: Maximal function characterizations for new local Hardy type spaces on spaces of homogeneous type. Trans. Am. Math. Soc. 370, 7229-7292 (2018). https://doi.org/10. 1090/tran/7289

8. Bui, H.-Q., Duong, X.T., Yan, L.: Calderón reproducing formulas and new Besov spaces associated with operators. Adv. Math. 229, 2449-2502 (2012)

9. Cacciafesta, F., D’Ancona, P.: Weighted $L^{p}$ estimates for powers of self-adjoint operators. Adv. Math. 229, 501-530 (2012)

10. Carron, G., Coulhon, T., Ouhabaz, E.M.: Gaussian estimates and $L^{p}$-boundedness of Riesz means. J. Evol. Equ. 2, 299-317 (2002)

11. Coifman, R.R., Weiss, G.: Extensions of Hardy spaces and their use in analysis. Bull. Am. Math. Soc. 83, 569-645 (1977)

12. Coulhon, T., Duong, X.T.: Maximal regularity and kernel bounds: observations on a theorem by Hieber and Prüss. Adv. Differ. Equ. 5(1-3), 343-368 (2000)

13. D'Ancona, P., Fanelli, L.: $L^{p}$-boundedness of the wave operator for the one dimensional Schrödinger operator. Commun. Math. Phys. 268, 415-438 (2006)

14. D'Ancona, P., Pierfelice, V.: On the wave equation with a large rough potential. J. Funct. Anal. 227(1), 30-77 (2005)

15. D'Ancona, P., Pierfelice, V., Ricci, F.: On the wave equation associated to the Hermite and the twisted Laplacian. J. Fourier Anal. Appl. 16(2), 294-310 (2010)

16. Deng, D., Duong, X.T., Sikora, A., Yan, L.: Comparison of the classical BMO with the BMO spaces associated with operators and applications. Rev. Mat. Iberoam. 24(1), 267-296 (2008)

17. Duong, X.T., Li, J.: Hardy spaces associated to operators satisfying Davies-Gaffney estimates and bounded holomorphic functional calculus. J. Funct. Anal. 264, 1409-1437 (2013)

18. Duong, X.T., Yan, L.: Duality of Hardy and BMO spaces associated with operators with heat kernel bounds. J. Am. Math. Soc. 18, 943-973 (2005)

19. Duong, X.T., Ouhabaz, E.M., Sikora, A.: Plancherel-type estimates and sharp spectral multipliers. J. Funct. Anal. 196, 443-485 (2002)

20. Dziubański, J.: Atomic decomposition of $H^{p}$ spaces associated with some Schrödinger operators. Indiana Univ. Math. J. 47, 75-98 (1998)

21. Frazier, M., Jawerth, B.: Decomposition of Besov spaces. Indiana Univ. Math. J. 34, 777-799 (1985)

22. Georgiev, V., Visciglia, N.: Decay estimates for the wave equation with potential. Commun. Partial Differ. Equ. 28(7-8), 1325-1369 (2003)

23. Ginibre, J., Velo, G.: Generalized Strichartz inequalities for the wave equation. J. Funct. Anal. 133(1), 50-68 (1995)

24. Guo, Z., Peng, L., Wang, B.: Decay estimates for a class of wave equations. J. Funct. Anal. 254, 1642-1660 (2008)

25. Hofmann, S., Lu, G., Mitrea, D., Mitrea, M., Yan, L.: Hardy spaces associated to non-negative selfadjoint operators satisfying Davies-Gaffney estimates. In: Memoirs of the American Mathematical Society, vol. 214 (2011)

26. Huang, T., Huang, S., Zheng, Q.: Inhomogeneous oscillatory integrals and global smoothing effects for dispersive equations (preprint). arXiv:1612.09072v1

27. Jiang, R., Yang, D.: Orlicz-Hardy spaces associated with operators sartisfying Davies-Gaffney estimates. Commun. Contemp. Math. 13, 331-373 (2011)

28. Journé, J.-L., Soffer, A., Sogge, C.D.: Decay estimates for Schrödinger operators. Commun. Pure Appl. Math. 44, 573-604 (1991)

29. Lebedev, N.N.: Special Functions and Their applications. Dover, New York (1972)

30. Marshall, B., Strauss, W., Wainger, S.: $L^{p}-L^{q}$ estimates for the Klein-Gordon equation. J. Math. Pures Appl. (9) 59(4), 417-440 (1980) 
31. Mauceri, G., Meda, S.: Equivalence of norms on finite linear combinations of atoms. Math. Z. 269, 253-260 (2011)

32. Müller, D., Ricci, F.: Analysis of second order differential operators on Heisenberg groups. I. Invent. Math. 101, 545-582 (1990)

33. Müller, D., Seeger, A.: Sharp $L^{p}$ bounds for the wave equation on groups of Heisenberg type. Anal. PDE 8(5), 1051-1100 (2015)

34. Ouhabaz, E.M.: Analysis of Heat Equations on Domains. London Mathematical Society Monographs Series, vol. 31. Princeton University Press, Princeton (2005)

35. Russ, E.: The atomic decomposition for tent spaces on spaces of homogeneous type. In: Asymptotic Geometric Analysis, Harmonic Analysis, and Related Topics, pp. 125-135, Proceedings of the Centre for Mathematical Analysis, Australian National University, vol. 42. Australian National University, Canberra (2007)

36. Simon, B.: Schrödinger semigroups. Bull. Am. Math. Soc. (N.S.) 7, 447-526 (1982)

37. Sohani, V.K.: Strichartz estimates for the Schrödinger propagator for the Laguerre operator. Proc. Indian Acad. Sci. (Math. Sci.) 123, 525-537 (2013)

38. Stein, E.M.: Harmonic Analysis: Real Variable Methods, Orthogonality and Oscillatory Integrals. Princeton University Press, Princeton (1993)

39. Thangavelu, S.: Lectures on Hermite and Laguerre Expansions. Princeton University Press, Princeton (1993)

40. Whitney, H.: Differentiable even functions. Duke Math. J. 10, 159-160 (1943)

41. Yajima, K.: The $W^{k, p}$-continuity of wave operators for Schrödinger operators. J. Math. Soc. Jpn. 47, 551-581 (1995)

42. Yajima, K.: $L^{p}$-boundedness of wave operators for two-dimensional Schrödinger operators. Commun. Math. Phys. 208, 125-152 (1999)

Publisher's Note Springer Nature remains neutral with regard to jurisdictional claims in published maps and institutional affiliations. 